Inra Prod. Anim., 2009, 22 (2), $117-130$

\section{Effet du bilan électrolytique de la ration sur l'équilibre acido-basique et les performances zootechniques des animaux domestiques à fort niveau de production}

\author{
E. APPER-BOSSARD I, J.-L. PEYRAUD ${ }^{2}$, J.-Y. DOURMAD 3 \\ ${ }^{1}$ Ecole Supérieure d'Agriculture, 22 rue Rabelais, F-49100 Angers, France \\ 2 INRA, Agrocampus, UMR1080 Production du lait, F-35590 Saint-Gilles, France \\ 3 INRA, Agrocampus, UMR1079 Systèmes d'Elevage, Nutrition Animale et Humaine, F-35590 Saint-Gilles, France \\ Courriel : e.bossard@groupe-esa.com
}

Directement absorbés lors de la digestion, les ions de la ration peuvent influencer l'homéostasie acido-basique et les performances zootechniques des animaux à fort potentiel de production.

\begin{abstract}
«La fixité du milieu intérieur est la condition d'une vie libre et indépendante» (Claude Bernard 1859).
\end{abstract}

L'homéostasie acido-basique est la capacité d'un animal à maintenir à niveau constant et adapté aux diverses réactions chimiques les paramètres physico-chimiques de l'organisme et notamment le $\mathrm{pH}$ grâce à des processus régulateurs variés. Les premiers principes ont été énoncés par Van Slyke (1922) qui a mis en évidence la constance du $\mathrm{pH}$ dans le sang, la lymphe et le liquide céphalorachidien et défini la notion de réserve alcaline. Très vite, l'homéostasie acido-basique est apparue comme une fonction vitale pour l'organisme soumis en permanence à des perturbations liées notamment à l'intensité du métabolisme cellulaire.

Les animaux à haut niveau de production ont une activité digestive et métabolique intense. Ils ont des besoins accrus par la fonction de production, besoins couverts par un niveau d'ingestion très élevé et des rations riches en énergie rapidement fermentescible. Or, chez le ruminant, l'énergie rapidement fermentescible est rapidement prise en charge par les bactéries du rumen pour être transformée en acides organiques, ce qui est à l'origine de baisse du $\mathrm{pH}$ ruminal, voire d'une baisse des réserves alcali- nes du sang (Faverdin et al 1999). Chez le monogastrique, l'énergie rapidement fermentescible est également rapidement digérée et transformée en acides organiques au niveau de l'intestin, pouvant entraînaer des baisses de $\mathrm{pH}$ sanguin. Du fait de ces perturbations acido-basiques, la maîtrise de l'alimentation est devenue un enjeu majeur. En effet, les dysfonctionnements d'origine digestive et métabolique sont à l'origine d'inefficacité zootechnique et finalement de pertes économiques. Ainsi, chez la vache laitière, l'acidose latente est aujourd'hui un problème très important en élevage. Peyraud et Apper-Bossard (2006) ont publié une revue sur cette pathologie en en présentant ses causes et les phénomènes métaboliques qu'elle implique. De même, chez le porc en croissance, une alimentation trop riche est responsable d'acidose métabolique qui, à terme, cause une perte d'appétit et donc une diminution du gain moyen quotidien (Patience et al 1987). Chez les ruminants comme chez les monogastriques, il est donc nécessaire de corriger les rations riches en énergie rapidement fermentescible, sources d'acidité, afin d'éviter ces problèmes.

Parmi les différentes pistes explorées, le contrôle du Bilan Electrolytique $(\mathrm{BE})$ de la ration, défini comme
$\mathrm{BE}=[$ sodium $]+[$ potassium $]-[$ chlore $]$ exprimé en $\mathrm{mEq} / \mathrm{kg}$ MS a souvent été évoqué. Sa première application a concerné l'élevage avicole afin de lutter contre la dyschondroplasie tibiale (Sauveur et Mongin 1978) ou encore pour améliorer la vitesse de croissance (Mongin 1981). Chez le porc en croissance, plusieurs essais ont été effectués afin d'étudier l'effet de l'accroissement du BE sur les performances zootechniques (Patience et al 1987, Dersjant-Li 2000). Chez la vache laitière, ce concept a d'abord servi dans la lutte contre la fièvre vitulaire (Jorgensen 1974). Ce sujet a d'ailleurs fait l'objet de nombreuses études nord-américaines depuis 30 ans. Plus récemment, des travaux ont été effectués pour analyser les effets du niveau de BE sur les performances zootechniques des vaches en pleine lactation (Coppock et al 1979, Takagi et Block 1991, Tucker et al 1994, ApperBossard 2005). Chez les porcs en croissance comme chez les vaches en lactation, une augmentation du BE de la ration semble être un levier d'action qui permet de limiter l'acidification du milieu extracellulaire. Néanmoins, aucune comparaison des lois de réponses physiologique et zootechnique à un accroissement du $\mathrm{BE}$ de la ration n'a été effectuée entre les ruminants et les monogastriques. C'est pourquoi, après avoir rappelé les bases de la 
régulation acido-basique des liquides extracellulaires et les mécanismes participant à l'homéostasie acidobasique, l'objectif de cet article est de décrire à partir d'une analyse quantitative de la bibliographie, les lois de réponse des paramètres de l'homéostasie acido-basique et des performances zootechniques face à des variations du $\mathrm{BE}$ des rations chez la vache laitière en pleine lactation, les bovins en croissance et les porcs en croissance.

\section{1 / Evaluation de l'homéo- stasie acido-basique du sang et mécanismes impli- qués dans son maintien}

Deux modèles principaux permettent de caractériser l'homéostasie acidobasique du sang et de décrire d'éventuelles perturbations : le modèle d'Henderson-Hasselbach et le modèle des ions forts.

La relation d'Henderson-Hasselbach caractérise l'homéostasie acidobasique en évaluant le $\mathrm{pH}$ et l'équilibre du système acide carbonique/ bicarbonate. Dans ce système, une mole de $\mathrm{CO}_{2}$ réagit avec une mole d'eau pour former de l'acide carbonique qui se dissocie ensuite pour former le bicarbonate. Cette réaction se fait essentiellement dans les hématies en présence d'anhydrase carbonique. Il s'agit du principal système tampon permettant de neutraliser la charge acide du sang. La relation d'Henderson-Hasselbach permet de définir la nature des déviations observées. Ces déviations sont l'acidose, lorsque le $\mathrm{pH}$ sanguin baisse, et l'alcalose si il augmente. Ces déviations sont liées à des désordres respiratoires ou métaboliques. Ainsi, l'acidose respiratoire se caractérise par une pression partielle en dioxyde de carbone $\left(\mathrm{P}_{\mathrm{CO} 2}\right)$ qui augmente; l'alcalose respiratoire se caractérise par une pression partielle en dioxyde de carbone qui diminue ; l'acidose métabolique se caractérise par des concentrations en bicarbonates du sang qui diminuent et l'alcalose métabolique par des concentrations en bicarbonates qui augmentent (Constable 1999). Les déviations métaboliques et respiratoires peuvent cohabiter chez un animal, d'où la nécessité de tenir compte des $\mathrm{pH}, \mathrm{P}_{\mathrm{CO} 2}$ et concentrations en bicarbonates simultanément (Davenport 1971). Pratiquement, le modèle d'HendersonHasselbach est un modèle descriptif qui permet de mettre en évidence des déviations éventuelles mais qui ne rend pas compte des phénomènes dynamiques et mécanistes mis en jeu.

Le deuxième modèle est celui des ions forts et constitue une approche plus mécaniste de l'évaluation de l'homéostasie acido-basique. Il a été présenté par Stewart $(1978,1983)$ qui a suggéré que le $\mathrm{pH}$ sanguin était déterminé par trois facteurs différents : i) la $\mathrm{P}_{\mathrm{CO} 2}$, ii) la charge en ions forts qui représente la différence en charge entre les cations et les anions complètement dissociés du plasma (appelée Différence en Ions Forts, DIFsang $=[$ sodium $]+[$ potassium $]-$ [chlore]) et iii) la concentration plasmatique totale en acides faibles non volatils (Atot) qui correspondent aux albumines, aux globulines et aux phosphates. Le pH est considéré comme une variable dépendante de la différence entre les charges positives et les charges négatives, et les réactions dans le plasma se réduisent à des réactions d'ions en solution du fait que les cations et les anions se lient sous forme de sels.

Les ions simples n'ont pas tous les mêmes propriétés. On distingue deux types distincts d'ions. Le premier type est celui des ions forts, sans pouvoir tampon et totalement dissociés aux $\mathrm{pH}$ physiologiques. Ils ont un pouvoir électrique et ne participent pas aux réactions dans le plasma. La composition du plasma en ions forts est exprimée par la DIF. Le second type regroupe les ions qui ont un pouvoir tampon. Ils dérivent des acides et bases faibles du plasma qui ne sont pas complètement dissociés aux $\mathrm{pH}$ physiologiques. Pour qu'un ion faible ait un pouvoir tampon significatif, son $\mathrm{pK}$ de dissociation doit se situer dans l'intervalle $\mathrm{pH} \pm 1,5$. Le $\mathrm{pH}$ sanguin étant de 7,40, les ions tampons dans le sang seront des ions dont le $\mathrm{pK}$ est compris entre 5,9 et 8,9 , ce qui est le cas des bicarbonates, des phosphates ou encore des groupements imidazoles des protéines. Afin de tenir compte des différentes molécules ayant un rôle tampon présentes dans le plasma, l'excès de bases est utilisé. Il s'agit d'une estimation plus précise de la composante métabolique que la seule mesure de la concentration en bicarbonates. Il se mesure par titration du sang avec un acide fort et prend en compte la contribution des tampons non volatils, notamment les concentrations en hémoglobine, en protéines et en phosphates du plasma.

\section{1 / Les mécanismes de régula- tion : systèmes tampons, respi- ration, diurèse et résorption osseuse}

L'organisme possède plusieurs types de mécanismes qui permettent de maintenir l'homéostasie acido-basique : les systèmes tampons, la respiration, la diurèse et la résorption osseuse. Les tampons sont des substances qui agissent immédiatement en captant les protons, limitant ainsi les baisses de $\mathrm{pH}$. Le principal système tampon du sang est le système acide carbonique/bicarbonate, puis les protéines plasmatiques et les globules rouges. La capacité tampon du sang est très limitée puisque les systèmes tampons captent les protons mais ne les éliminent pas. Par ailleurs, le fonctionnement du système acide carbonique/ bicarbonate engendre la formation de dioxyde de carbone dans le sang qui doit ensuite être éliminé. Il existe deux grands mécanismes d'élimination des protons et du gaz carbonique : la respiration et la diurèse.

La respiration est une fonction essentielle puisqu'elle permet l'acheminement du dioxyde de carbone tissulaire issu du métabolisme cellulaire, jusqu'aux poumons, où il est évacué. C'est le mécanisme de régulation de l'homéostasie acido-basique le plus efficace quantitativement, puisqu'il permet l'évacuation de $13000 \mathrm{mEq}$ de dioxyde de carbone par jour chez le porc (Brugère 2001). La respiration joue un rôle prépondérant dans le maintien de l'homéostasie acidobasique grâce à la ventilation. La $\mathrm{P}_{\mathrm{CO} 2}$ est un critère qui rend compte de la composante respiratoire de l'équilibre acido-basique. Elle est le résultat des échanges entre l'air contenu dans les alvéoles et le dioxyde de carbone apporté par le sang (Shapiro et al 1992). La $\mathrm{P}_{\mathrm{CO} 2}$ fait donc partie des composantes à mesurer lors de l'évaluation de l'équilibre acido-basique d'un animal. Par exemple, en cas d'accumulation de dioxyde de carbone dans le sang, le $\mathrm{pH}$ sanguin diminue tandis que la $\mathrm{P}_{\mathrm{CO} 2}$ augmente, témoignant d'une acidose respiratoire. La respiration est un mécanisme très efficace pour éliminer les charges de dioxyde de carbone.

La diurèse a quant à elle deux fonctions principales : l'excrétion d'acides et la régénération des bicarbonates. La réabsorption des bicarbonates a lieu dans le tubule contourné proximal et implique le sodium, le potassium et l'anhydrase carbonique qui 
transforme le dioxyde de carbone en bicarbonates par hydratation (Block 1995). Le rein permet donc d'enrichir le plasma en bicarbonates, ce qui lui confère un rôle de premier plan dans la régulation de l'équilibre acidobasique.

La résorption osseuse est également un mécanisme de régulation de l'équilibre acido-basique dans le long terme. En effet, le squelette constitue un réservoir de bases qui peut jouer le rôle de tampon sanguin lorsque le rein ne peut plus maintenir l'homéostasie acido-basique. Des études menées in vivo chez la souris mises en situation d'acidose métabolique ont montré qu'il y avait une perte du sodium et du potassium osseux, ainsi qu'une déplétion en bicarbonates et en phosphates (Bushinsky et al 1999, Bushinsky et al 2003, Krieger et al 2003). Des études menées in vitro ont parallèlement montré une augmentation du nombre de cellules ostéoclastiques (Meghji et al 2001) ou une chute du nombre de cellules ostéoblastiques (Frick et Bushinsky 1998). L'hormone parathyroïdienne semble réguler l'intensité de la résorption osseuse en fonction des besoins en tampons du sang : en cas d'acidose ou d'hypocalcémie, le taux circulant de cette hormone s'accroît, stimulant la production surrénale de la 1,25-dihydroxycholecalciferol (Goff et al 1991). Cette hormone active ensuite les enzymes lysosomales $\mathrm{pH}$ dépendantes dans les ostéoclastes (Block 1995).

L'organisme possède un ensemble de mécanismes impliqués dans la régulation de l'équilibre acidobasique. Ces mécanismes ont des actions qui permettent de compenser des variations d'acidité à plus ou moins long terme. Néanmoins, malgré ces différents systèmes de régulation, les perturbations ne sont pas toujours entièrement compensées et peuvent entraîner des maladies métaboliques. Parmi elles, les plus fréquentes chez les animaux d'élevage sont l'acidose métabolique, liée à une chute du $\mathrm{pH}$ sanguin et des concentrations en bicarbonates et l'acidose respiratoire, liée à une chute du $\mathrm{pH}$ sanguin et une augmentation de la $\mathrm{P}_{\mathrm{CO} 2}$. Chez le ruminant, l'acidose métabolique est moins fréquente que chez le monogastrique du fait du rumen qui prend en charge une bonne partie de la production des acides. Par conséquent, le $\mathrm{pH}$ sanguin chute moins rapidement que chez un monogastrique, tandis que le $\mathrm{pH}$ du rumen peut chuter, entraînant une acidose ruminale. L'ensemble de ces perturbations est d'autant plus fréquent que les animaux ont un niveau élevé de production et d'ingestion. Il apparaît donc nécessaire de limiter les risques de perturbations acidobasiques liées à l'alimentation.

\section{2 / Intérêt de la manipulation des teneurs en ions forts de la ration}

L'idée de manipuler les ions forts de la ration pour la sécuriser découle des connaissances détenues en physiologie : les cations amenés par la ration sont directement échangés avec des protons lors de l'absorption digestive, permettant l'augmentation du $\mathrm{pH}$ sanguin d'après le modèle des ions forts établi par Stewart (1978, 1983). Ainsi, une ration au BE élevé apporte beaucoup de cations échangés avec les protons. Dans une ration riche en énergie rapidement fermentescible, un $\mathrm{BE}$ élevé permet donc de compenser la charge acide et permet au $\mathrm{pH}$ sanguin de se maintenir.

Les ions les plus utilisés pour manipuler la ration sont le sodium, le potassium et le chlore. Plusieurs raisons argumentent ce choix : d'abord, ils sont quasi totalement absorbables quelle que soit la source alimentaire (Meschy et Guéguen 1998). Ensuite, ce sont des constituants essentiels du sang. Le sodium et le potassium sont échangés contre des protons, le chlore contre des bicarbonates dans de nombreux processus métaboliques afin de conserver l'électroneutralité des tissus (Constable 1999). La ration est alors caractérisée par la différence [sodium] + [potassium] - [chlore]. L'inclusion du soufre dans le calcul de cette différence est parfois effectuée, mais est encore discutée. Elle est argumentée par le fait que le soufre possède la capacité d'altérer l'homéostasie acido-basique des organismes (Oetzel et al 1991, Block 1994). Cependant, le soufre qui possède un pouvoir acidifiant inférieur à celui du chlore, est difficile et long à doser, et sa disponibilité dans les fourrages dépend de la digestibilité des fractions azotées. Au final, le soufre n'a pas été pris en compte dans la totalité des études réalisées ou a été pris en considération avec des facteurs de pondération différents d'une étude à l'autre. C'est le bilan électrolytique $(\mathrm{BE}=[$ sodium $]+[$ potassium $]$ [chlore]) qui a été retenu pour réaliser cette analyse.

\section{2 / Quantification de l'effet de la composition en ions de la ration sur l'homéostasie acido-basique des monogas- triques et des ruminants}

\section{1 / Constitution de la base de données}

Afin d'établir les lois de réponse, trois bases de données ont été constituées : une première pour les vaches en lactation, une seconde pour les ruminants en croissance et finition et une troisième pour les porcs en croissance et finition. Pour être retenues, les publications devaient fournir les teneurs en sodium, en potassium et en chlore, tester au moins deux niveaux du facteur et fournir au moins le $\mathrm{pH}$, la $\mathrm{P}_{\mathrm{CO} 2}$ et les concentrations en bicarbonates du sang. Les animaux devaient par ailleurs être alimentés à volonté. En outre, les publications devaient fournir si possible les teneurs en minéraux du sang et le $\mathrm{pH}$ urinaire.

Chez les vaches laitières, 14 publications ont été retenues, correspondant à 61 traitements et 250 observations (Coppock et al 1979, Kilmer et al 1980, Escobosa et al 1984, Tucker et al 1988a, 1988b, McKinnon et al 1990, Tucker et Hogue 1990, Tucker et al 1991a, 1994, Waterman et al 1991, West et al 1991, 1992, Delaquis et Block 1995a, Delaquis et Block 1995b). Les BE ont varié de - 191 à $+364 \mathrm{mEq} / \mathrm{kg} \mathrm{MS}$, le $\mathrm{pH}$ sanguin de 7,31 à 7,50, les teneurs en bicarbonates de 18,7 à $31,0 \mathrm{mEq} / \mathrm{L}$ et la $\mathrm{P}_{\mathrm{CO} 2}$ de 32,0 à $51,5 \mathrm{mmHg}$. Dans tous les essais, les vaches ont reçu des rations complètes avec une teneur en concentré qui a varié de 40 à $70 \%$ (tableau 1). Les sels utilisés pour augmenter le BE étaient le bicarbonate de sodium (10 publications), le bicarbonate de potassium (4 publications), le sesquicarbonate de sodium (1 publication) et le carbonate de potassium (1 publication). Les sels utilisés pour diminuer le $\mathrm{BE}$ étaient le chlorure de calcium (6 publications).

Chez les ruminants en croissance, 10 publications ont été retenues, correspondant à 37 traitements. Deux publications avaient comme espèce modèle des moutons (Takagi et Block 1991, Fauchon et al 1995), cinq des veaux sevrés, âgés d'au moins 6 semaines (Wamberg et al 1985, Beighle et al 1988, Den Hartog et al 1989, Tucker et al 1991b, Jackson et al 1992), deux des bouvillons (Ross et 
Tableau 1. Moyenne et amplitude du BE de la ration et des différentes variables testées dans notre base de données sur les vaches en lactation.

\begin{tabular}{|c|c|c|c|c|c|}
\hline & Nombre & Moyenne & Ecart-type & Minimum & Maximum \\
\hline Ration & 61 & 300 & & 101 & 624 \\
\hline $\begin{array}{l}\mathrm{BE}, \mathrm{m} \in \mathrm{q} / \mathrm{kg} \mathrm{MS} \\
\text { Concentré, \% MS }\end{array}$ & $\begin{array}{l}61 \\
61\end{array}$ & $\begin{array}{l}309 \\
55,8\end{array}$ & $\begin{array}{c}182,4 \\
7,89\end{array}$ & $\begin{array}{l}-191 \\
40,0\end{array}$ & $\begin{array}{l}634 \\
70,0\end{array}$ \\
\hline $\mathrm{MAT}^{2}, \% \mathrm{MS}$ & 61 & 16,9 & 1,36 & 14,8 & 19,1 \\
\hline $\begin{array}{l}\mathrm{MSI}^{3}, \mathrm{~kg} / \mathrm{j} \\
\text { Lait }\end{array}$ & 55 & 18,2 & 2,15 & 12,6 & 23,6 \\
\hline Production, kg/j & 55 & 23,8 & 5,98 & 14,9 & 41,0 \\
\hline Lait $4 \%, \mathrm{~kg} / \mathrm{j}$ & 53 & & & & \\
\hline Taux Protéique, $\mathrm{g} / \mathrm{kg}$ & 53 & 32,3 & 1,55 & 27,7 & 35,0 \\
\hline Taux Butyreux, $\mathrm{g} / \mathrm{kg}$ & 53 & 36,4 & 4,72 & 26,0 & 44,1 \\
\hline Matières protéiques, $\mathrm{g} / \mathrm{j}$ & 53 & 764 & 189,6 & 510 & 1220 \\
\hline $\begin{array}{l}\text { Matières grasses, } g / j \\
\text { Sang }\end{array}$ & 53 & 846 & 176,6 & 582 & 1410 \\
\hline $\mathrm{pH}$ & 59 & 7,41 & 0,040 & 7,31 & 7,50 \\
\hline $\mathrm{HCO}_{3}{ }^{-}, \mathrm{mEq} / \mathrm{L}$ & 51 & 26,3 & 3,24 & 18,7 & 31,0 \\
\hline $\mathrm{P}_{\mathrm{CO} 2}, \mathrm{mmHg}$ & 51 & 42,0 & 5,33 & 32,0 & 51,5 \\
\hline $\mathrm{Na}^{+}, \mathrm{mEq} / \mathrm{L}^{-}$ & 49 & 143 & 6,56 & 124 & 162 \\
\hline $\mathrm{K}^{+}, \mathrm{mEq} / \mathrm{L}$ & 49 & 4,88 & 0,920 & 3,65 & 7,30 \\
\hline $\mathrm{Cl}^{-}, \mathrm{mEq} / \mathrm{L}$ & 49 & 102 & 3,92 & 87,4 & 109 \\
\hline $\begin{array}{l}\mathrm{DIF}^{4} \text { sang, } \mathrm{mEq} / \mathrm{L} \\
\text { Urine }\end{array}$ & 49 & 46,2 & 6,32 & 37,5 & 68,0 \\
\hline $\mathrm{pH}$ & 55 & 7,46 & 0,930 & 5,41 & 8,65 \\
\hline
\end{tabular}

${ }^{1} \mathrm{BE}$ ration $=$ Bilan Electrolytique de la ration, $\mathrm{BE}=\mathrm{Na}^{+}+\mathrm{K}^{+}-\mathrm{Cl}^{-}$.

2MAT : Matières Azotées Totales.

${ }^{3} \mathrm{MSI}$ : Matière Sèche Ingérée.

${ }^{4} \mathrm{DIF}=$ Différence en lons Forts du sang, $\mathrm{DIF}=\mathrm{Na}^{+}+\mathrm{K}^{+}-\mathrm{Cl}^{-}$.

al 1994a, 1994b) et un des génisses (La Manna et al 1999). Les BE ont varié de -4 à $+700 \mathrm{mEq} / \mathrm{kg} \mathrm{MS}$, le $\mathrm{pH}$ sanguin de 7,34 à 7,47, les teneurs en bicarbonates de 19,8 à $31 \mathrm{mEq} / \mathrm{L}$ et la $\mathrm{P}_{\mathrm{CO} 2}$ de 29,7 à $48,7 \mathrm{mmHg}$ (tableau 2). Les sels utilisés pour aug-

menter le BE étaient les bicarbonates de sodium ( 9 articles) et de potassium (1 article) ainsi que du carbonate de sodium (1 article). Les sels utilisés pour diminuer le BE étaient le chlorure de calcium ( 2 articles) et le chlorure d'ammonium (4 articles).

Tableau 2. Moyenne et amplitude du BE de la ration et des différentes variables testées dans notre base de données sur les ruminants en croissance.

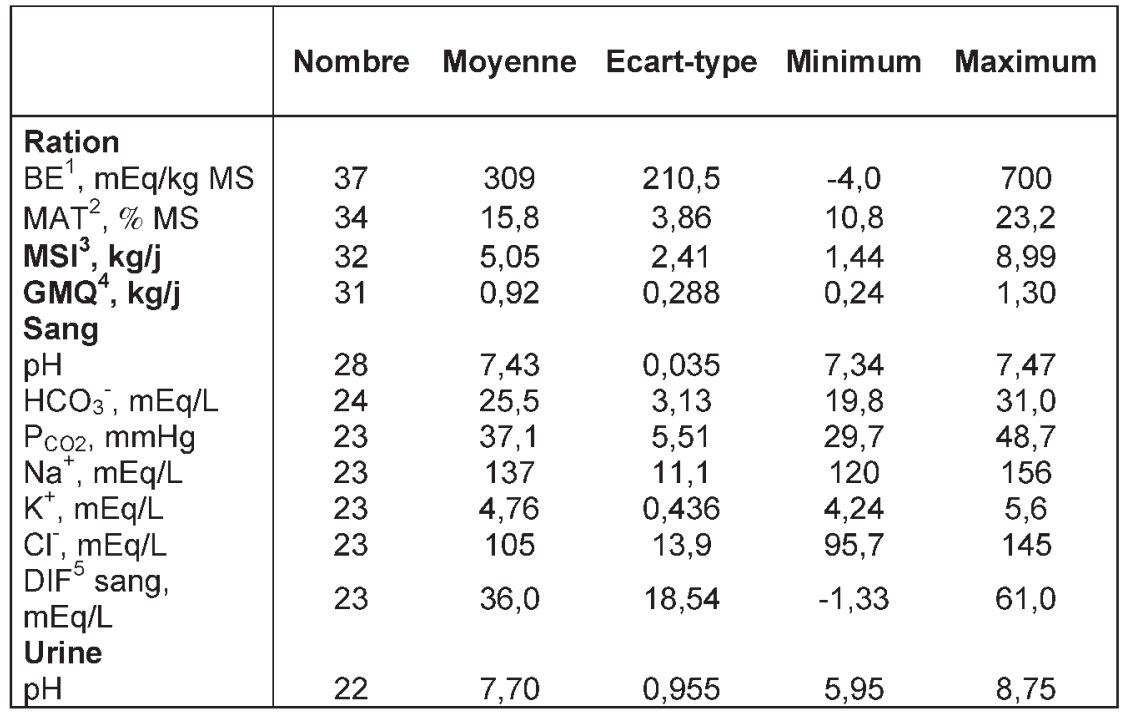

${ }^{1} \mathrm{BE}$ ration $=$ Bilan Electrolytique de la ration, $\mathrm{BE}=\mathrm{Na}^{+}+\mathrm{K}^{+}-\mathrm{Cl}^{-}$.

2MAT : Matières Azotées Totales.

$3 \mathrm{MSI}$ : Matière Sèche Ingérée.

${ }^{4} \mathrm{GMQ}=$ Gain Moyen Quotidien.
Chez le porc, sept publications ont été retenues, correspondant à 63 traitements (Honeyfield et Froseth 1985, Patience et al 1987, Haydon et al 1990 Patience et Wolynetz 1990, Wondra et al 1995, Patience et Chaplin 1997, Dersjant-Li 2000). Les BE ont varié de - 181 à $+510 \mathrm{mEq} / \mathrm{kg} \mathrm{MS}$, le $\mathrm{pH}$ sanguin de 7,04 à 7,48 , les teneurs en bicarbonates de 21,0 à $36,5 \mathrm{mEq} / \mathrm{L}$ et la $\mathrm{P}_{\mathrm{CO} 2}$ de 36,7 à 94 mmHg (tableau 3). Les rations étaient à base de maïs grain (6 auteurs), ou d'orge (1 auteur) à raison de $80 \%$ de la MS. Les sels utilisés pour augmenter le BE étaient les bicarbonates de sodium ( 7 articles) et de potassium (1 article). Les sels utilisés pour diminuer le BE étaient le chlorure de calcium (3 articles).

Les données zootechniques et physiologiques ont été analysées par régression linéaire en prenant le $\mathrm{BE}$ pour tester une réponse linéaire et le $\mathrm{BE}$ élevé au carré pour tester une éventuelle réponse quadratique. Un effet fixe lié à la publication a également été considéré. Lorsque la réponse quadratique n'était pas significative, l'analyse était refaite avec le BE de la ration comme seule covariable. Les variables testées ont été le $\mathrm{pH}$, la $\mathrm{P}_{\mathrm{CO} 2}$, les concentrations en bicarbonates, le BE du sang et les concentrations en chlore, en potassium et en sodium du sang.

\section{2 / Caractéristiques de l'ho- méostasie acido-basique}

Les paramètres de l'homéostasie acido-basique sont très semblables entre les vaches laitières et les ruminants en croissance, avec un $\mathrm{pH}$ moyen de 7,42 et des concentrations en bicarbonates proches de $26 \mathrm{mEq} / \mathrm{L}$. Seule la $\mathrm{P}_{\mathrm{CO} 2}$ est plus élevée chez les vaches laitières (42 vs $37 \mathrm{mmHg}$, tableau 4). Les porcs sont caractérisés par un $\mathrm{pH}$ sanguin plus faible $(7,22)$ et surtout une $\mathrm{P}_{\mathrm{CO} 2}$ très élevée $(73 \mathrm{mmHg}$ ) comparativement aux ruminants. De telles valeurs traduisent un profil d'acidose respiratoire marquée. Les teneurs en bicarbonates sont légèrement plus élevées chez les porcs que chez les ruminants (29 vs $26 \mathrm{mEq} / \mathrm{L})$.

Le $\mathrm{pH}$ sanguin est dans l'ensemble relativement stable, confirmant l'existence de mécanismes de régulation de la concentration en protons très efficaces. Néanmoins, après corrections des effets publications, une variation d'environ 0,15 unités $\mathrm{pH}$ est observée chez les porcs comme chez les ruminants. Les teneurs en bicarbonates du sang varient dans des proportions plus larges 
Tableau 3. Moyenne et amplitude du BE de la ration et des différentes variables testées dans notre base de données sur les porcs en croissance.

\begin{tabular}{|l|ccccc|}
\hline & Nombre & Moyenne & Ecart-type & Minimum & Maximum \\
\hline Ration & & & & & \\
$\mathrm{BE}^{1}, \mathrm{mEq} / \mathrm{kg} \mathrm{MS}$ & 63 & 149 & 145,7 & -181 & 510 \\
$\mathrm{MSI}^{2}, \mathbf{k g} / \mathrm{j}$ & 58 & 1,47 & 0,642 & 0,58 & 2,95 \\
$\mathrm{GMQ}^{3}, \mathbf{k g} / \mathrm{j}$ & 58 & 0,59 & 0,207 & 0,14 & 0,92 \\
$\mathrm{Sang}$ & & & & & \\
$\mathrm{pH}$ & 52 & 7,22 & 0,091 & 7,04 & 7,48 \\
$\mathrm{HCO}{ }_{3}^{-}, \mathrm{mEq} / \mathrm{L}$ & 52 & 28,8 & 3,50 & 21,0 & 36,5 \\
$\mathrm{PCO}_{\mathrm{mmHg}}$ & 46 & 73,0 & 12,0 & 36,7 & 94,0 \\
$\mathrm{Na}^{+}, \mathrm{mEq} / \mathrm{L}$ & 30 & 141 & 7,57 & 124 & 154 \\
$\mathrm{~K}^{+}, \mathrm{mEq} / \mathrm{L}$ & 30 & 6,1 & 1,26 & 3,9 & 8,0 \\
$\mathrm{Cl}^{-}, \mathrm{mEq} / \mathrm{L}$ & 30 & 102 & 4,62 & 85 & 110 \\
$\mathrm{DIF}^{4}$ sang, $\mathrm{mEq} / \mathrm{L}$ & 30 & 49,0 & 4,85 & 40,0 & 59,0 \\
\hline
\end{tabular}

${ }^{1} \mathrm{BE}$ ration $=$ Bilan Electrolytique de la ration, $\mathrm{BE}=\mathrm{Na}^{+}+\mathrm{K}^{+}-\mathrm{Cl}^{-}$.

2MSI : Matière Sèche Ingérée.

3GMQ = Gain Moyen Quotidien.

${ }^{4} \mathrm{DIF}=$ Différence en lons Forts du sang, DIF $=\mathrm{Na}^{+}+\mathrm{K}^{+}-\mathrm{Cl}^{-}$.

(8 à $9 \mathrm{mEq} / \mathrm{L}$ ), de même que la $\mathrm{P}_{\mathrm{CO} 2}$ (4 à $10 \mathrm{mmHg}$ ).

La natrémie a été très semblable entre les vaches laitières et les porcs (143 et $141 \mathrm{mEq} / \mathrm{L}$ respectivement) et un peu faible chez les ruminants en croissance $(137 \mathrm{mEq} / \mathrm{L})$. La kaliémie est très semblable entre tous les ruminants $(4,7 \mathrm{mEq} / \mathrm{L}$ en moyenne) et sensiblement plus élevée chez les porcs $(6,1 \mathrm{mEq} / \mathrm{L})$. La chlorémie est peu variable entre espèces. Elle a été de $103 \mathrm{mEq} / \mathrm{L}$ en moyenne. Au final, la différence en ions forts du sang est plus élevée chez les porcs, intermédiaire chez les vaches et un peu plus faible chez les ruminants en croissance.

\section{3 / Les réponses de l'homéos- tasie acido-basique aux varia- tions du BE des rations sont cohérentes entre ruminants et monogastriques}

Le tableau 4 présente les différents coefficients de régression entre le $\mathrm{BE}$ de la ration et les différentes variables de l'homéostasie acido-basique testées obtenus dans les 3 modèles.

Le $\mathrm{pH}$ sanguin et les concentrations en bicarbonates augmentent quadratiquement avec l'augmentation du BE de la ration chez tous les ruminants et chez les porcs (figures 1 et 2). Selon les équations d'ajustement obtenues, un accroissement du BE de 0 à $+200 \mathrm{mEq} / \mathrm{kg}$ MS correspond à un accroissement du $\mathrm{pH}$ sanguin de 7,37 à 7,40 pour les vaches laitières, de 7,40 à 7,42 pour les ruminants en croissance et de 7,20 à 7,25 pour les porcs. Ce même accroissement du $\mathrm{BE}$ de la ration se traduit par une augmentation des concentrations en bicarbonates du sang de 23,4 à $25,6 \mathrm{mEq} / \mathrm{L}$ chez la vache laitière, de 22,8 à 25,1 chez les ruminants en croissance et de 26,8 à 30,1 chez les porcs.

Le $\mathrm{pH}$ et les concentrations en bicarbonates sanguins augmentent très rapidement pour des BE compris entre - 200 et + $100 \mathrm{mEq} / \mathrm{kg} \mathrm{MS}$, atteignent un maximum pour des $\mathrm{BE}$ de la ration proches de $350 \mathrm{mEq} / \mathrm{kg}$ MS pour ensuite peu varier pour des BE compris entre 300 et $500 \mathrm{mEq} / \mathrm{kg}$ MS. De tels effets ont déjà été décrits chez le cheval (Mueller et al 1999), le poulet (Mongin 1981, Whitehead 1997, Murakami et al 2001) et le chat (Ching et al 1989), mais les données sur ces espèces étaient trop rares pour réaliser une approche quantitative.

Chez tous les ruminants, le $\mathrm{pH}$ urinaire augmente fortement et de manière quadratique avec le $\mathrm{BE}$ de la ration (figure 3). La loi de réponse du $\mathrm{pH}$ urinaire est très semblable entre les deux types de modèles «ruminants». Selon les lois de réponse calculées, un accroissement du $\mathrm{BE}$ de la ration de - 200 à $+400 \mathrm{mEq} / \mathrm{Kg}$ MS se traduit par un accroissement du $\mathrm{pH}$ urinaire de 3,6 unités en moyenne. Le $\mathrm{pH}$ de l'urine est maximal pour des $\mathrm{BE}$ de la ration

Tableau 4. Coefficients de régression entre le $B E$ de la ration $\left(B E=\mathrm{Na}^{+}+\mathrm{K}^{+}-\mathrm{Cl}^{-}\right)$et les différentes variables de l'homéostasie acidobasique testées obtenus dans les 3 modèles.

\begin{tabular}{|c|c|c|c|c|c|c|c|c|c|}
\hline \multirow[b]{2}{*}{ Variable } & \multicolumn{3}{|c|}{$\begin{array}{c}\text { Vaches } \\
\text { en lactation }\end{array}$} & \multicolumn{3}{|c|}{$\begin{array}{l}\text { Ruminants } \\
\text { en croissance }\end{array}$} & \multicolumn{3}{|c|}{$\begin{array}{c}\text { Porcs } \\
\text { en croissance-finition }\end{array}$} \\
\hline & $\mathrm{BE}=0$ & $\mathrm{~L}^{2}$ & $Q^{3}$ & $B E=0$ & $\mathrm{~L}^{2}$ & $Q^{3}$ & $\mathrm{BE}=0$ & $\mathrm{~L}^{2}$ & $Q^{3}$ \\
\hline Sang & & & & & & & & & \\
\hline $\mathrm{pH}$ & 7,368 & 0,00023 & $-2,7.10^{-1}$ & 7,399 & 0,00013 & $-9,3.10^{-8^{*}}$ & 7,204 & 0,00028 & $-3,5.10^{-7}$ \\
\hline $\begin{array}{l}\text { 3, mEq/L } \\
\mathrm{mmHa}\end{array}$ & 23,4 & 0,0134 & $-1,2.10$ & 22,8 & 0,0142 & $-1,3.100$ & 26,8 & 0.0252 ** & $-4,3.10$ \\
\hline $\mathrm{Na}^{+}, \mathrm{mEq} / \mathrm{L}$ & 41,0 & NS & NS & 30,2 & NS & NS & 10.0 & NS & NS \\
\hline $\mathrm{K}^{+}, \mathrm{mEq} / \mathrm{L}$ & & NS & NS & & NS & NS & & NS & NS \\
\hline $\mathrm{Cl}^{-}, \mathrm{mEq} / \mathrm{L}$ & 104,4 & $-0,0105^{* *}$ & NS & 108,7 & $-0,0077^{\star *}$ & NS & 103,4 & $0,0096^{\dagger}$ & NS \\
\hline $\mathrm{DIF}^{1}, \mathrm{mEq} / \mathrm{L}$ & 42,0 & $0,0164^{\star \star \star}$ & NS & 31,8 & $0,0106^{\star *}$ & NS & 47,1 & $0,0122^{*}$ & NS \\
\hline $\begin{array}{l}\text { Urine } \\
\mathrm{pH}\end{array}$ & 5,84 & $0,0068^{* * *}$ & $-4,1.10^{-6^{*}}$ & 6,09 & $0,00729^{* * * *}$ & $-5,4 \cdot 10^{-6^{+\ldots+1}}$ & - & - & - \\
\hline
\end{tabular}

\footnotetext{
$2 \mathrm{~L}=$ effet linéaire

${ }^{3} \mathrm{Q}=$ effet quadratique-

$\dagger P<0,10$

${ }^{*} \mathrm{P}<0,05$

${ }^{* *} \mathrm{P}<0,01$

${ }^{* * *} \mathrm{P}<0,001$
}

${ }^{4} \mathrm{DIF}=$ Différence en lons Forts du sang, $\mathrm{DIF}=\mathrm{Na}^{+}+\mathrm{K}^{+}-\mathrm{C}$ 
Figure 1. Effet de l'accroissement du BE de la ration sur le $\mathrm{pH}$ sanguin des vaches en lactation $(\boldsymbol{A}$. $\mathrm{pH}$ sanguin $=7,368+0,00023$ ' $\left.B E-2,73^{\prime} 10^{-7}{ }^{\prime} B E^{2}, r^{2}=0,95, E T R=0,0107, P<0,001, n=59.\right)$, des ruminants en croissance $(B$. $p H$ sanguin $=7,399+0,00013$ $\left.B E-9,3^{\prime} 10^{-8} \cdot B E^{2}, r^{2}=0,92, E T R=0,0120, P<0,02, n=28\right)$ et des porcs en croissance et finition (C. $p H$ sanguin $=7,204+$ $0,00028^{\prime} B E-3,5^{\prime} 10^{-7} \cdot B E^{2}, r^{2}=0,96, E T R=0,019, P<0,01, \mathrm{n}=52$ ).

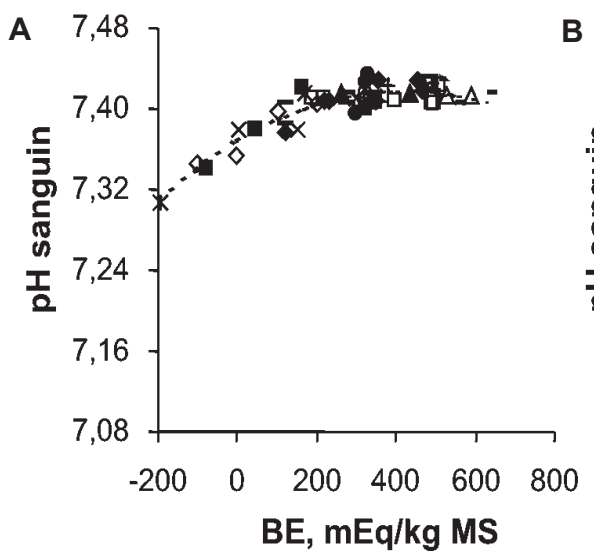

\footnotetext{
$\triangle$ Delaquis et Block 1995a + Delaquis et Block 1995b * Escobosa et al $1984 \quad$ Kilmer et al 1980

- McKinnon et al $1990 \diamond$ Tucker et al 1988a

- Tucker et al 1988b

口 Tucker et al 1991a et b $\Delta$ Tucker et al 1994

$\times$ Waterman et al 1991 West et al 1992

West et al 1991
}

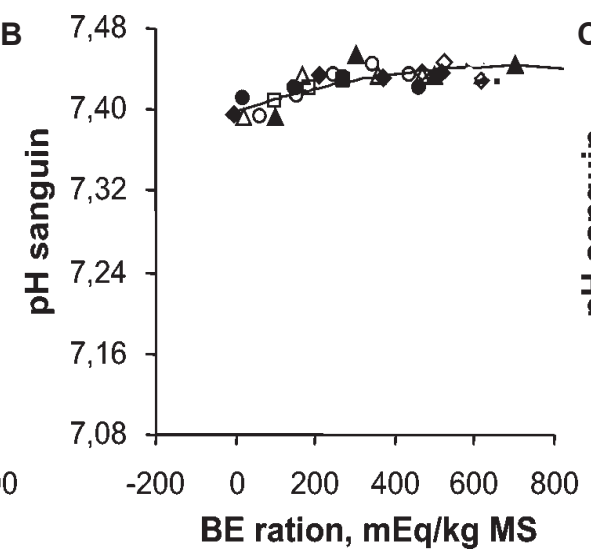

口 La Manna et al 1999

A Fauchon et al 1995

- Jackson et al 1992

$\triangle$ Ross et al 1994a

- Ross et al 1994b

$\diamond$ Wamberg et al 1991

o Den Hartog et al 1989 à $750 \mathrm{mEq} / \mathrm{kg} \mathrm{MS}$. En fait, le $\mathrm{pH}$ urinaire ne varie pratiquement plus pour des BE supérieurs à $500 \mathrm{mEq} / \mathrm{Kg} \mathrm{MS}$. Une autre analyse quantitative (Spanghero 2004) a mis en évidence qu'il existait une corrélation étroite entre le $\mathrm{BE}$ de la ration et le $\mathrm{pH}$ urinaire des vaches prepartum. Nos données et celles de Spanghero (2004) suggèrent que la relation est valable quel que soit le stade physiologique du ruminant. Plusieurs essais rapportent également une augmentation du $\mathrm{pH}$ urinaire lorsque le BE de la ration s'accroît chez le porc (Patience et Chaplin 1997) mais la loi de réponse n'a pas pu être calculée du fait du faible nombre de don- nées. D'autres travaux rapportent aussi une liaison positive entre le $\mathrm{pH}$ urinaire et le BE de la ration chez le cheval (Wall et al 1992, Mueller et al 1999) et la chèvre en lactation (Freeden et al 1988).

La relation forte existant entre le $\mathrm{pH}$ urinaire et le $\mathrm{BE}$ de la ration établit un lien physiologique entre l'ingestion des minéraux et leur excrétion et confirme que la diurèse est le mécanisme primordial dans la régulation des flux d'ions forts. Concrètement, les animaux ont excrété plus de protons avec des rations au $\mathrm{BE}$ faible. Cette relation a été montrée également chez

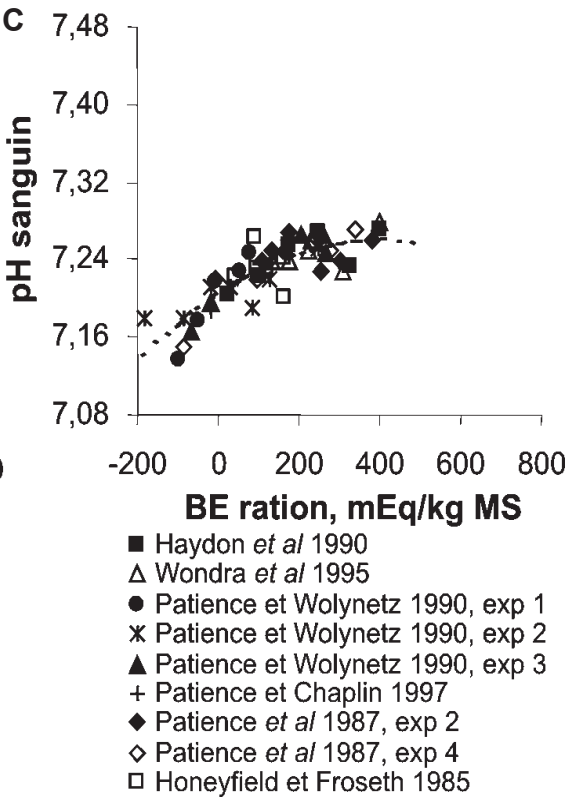

les vaches prepartum (Spanghero 2004). Par ailleurs, cette relation est très stable dans le temps et le $\mathrm{pH}$ urinaire n'est pas sensible à la variation de la teneur en énergie rapidement fermentescible de la ration (ApperBossard 2005) si bien que le $\mathrm{pH}$ urinaire pourrait être utilisé comme indicateur du BE de la ration, actuellement difficile à estimer sans faire de dosage des aliments.

L'ensemble des lois de réponse décrites tant chez les monogastriques que chez les ruminants confirme le rôle majeur des ions forts apportés par la ration sur le maintien de l'homéostasie

Figure 2. Effet de l'accroissement du $\mathrm{BE}$ de la ration sur les concentrations en $\mathrm{HCO}_{3}{ }^{-}$du sang des vaches en lactation $\left(\boldsymbol{A}\right.$. [HCO $\left.\mathrm{HCO}_{3}\right]$ $\left.=23,38+0,0134^{\prime} B E-1,23^{\prime} 10^{-5} \cdot B E^{2}, r^{2}=0,95, E T R=0,811, P<0,01, n=51\right)$, des ruminants en croissance $\left(B\right.$. $\left[H_{C O}{ }^{-}\right]=$ $\left.22,80+0,0142^{\prime} B E-1,30^{\prime} 10^{-5}{ }^{\prime} B^{2}, r^{2}=0,94, E T R=0,945, P<0,05, n=24\right)$ et des porcs en croissance et finition $\left(C^{C}\right.$. [HCO $\left.{ }_{3}^{-}\right]$ $=26,76+0,0252^{\prime} B E-4,30^{\prime} 10^{-5} \cdot B E^{2}, r^{2}=0,95, E T R=0,859, P=0,0001, \mathrm{n}=52$ ).
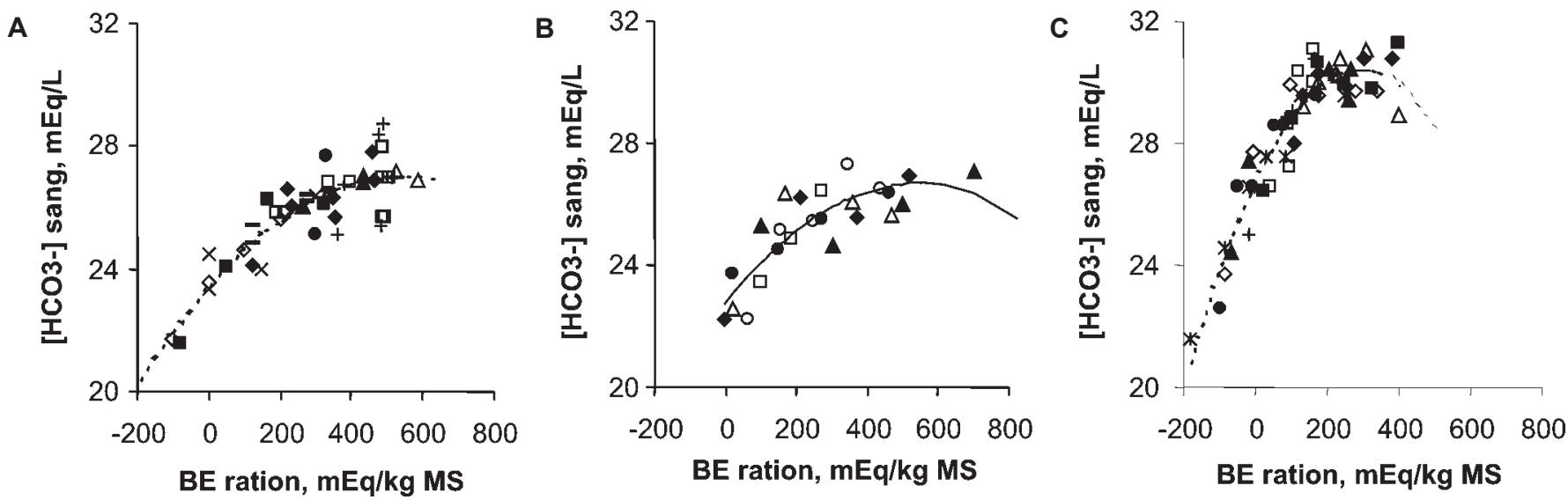
Figure 3. Effet de l'accroissement du BE de la ration sur le $\mathrm{pH}$ urinaire des vaches en lactation $\left(\boldsymbol{A}\right.$. $\mathrm{pH}$ urinaire $=5,836+0,0068^{\prime} \mathrm{BE}-4,09^{\prime} 10^{-6} \mathrm{~B}^{\prime} \mathrm{BE}^{2}, \mathrm{r}^{2}=0,88, \mathrm{ETR}=$ $0,369, P<0,02, \mathrm{n}=55$ ) et des ruminants en croissance (B. $\mathrm{pH}$ urinaire $=6,094+$ $\left.0,00729^{\prime} B E-5,4^{\prime} 10^{-6} \cdot B E^{2}, r^{2}=0,92, E T R=0,330, P<0,001, \mathrm{n}=22\right)$.

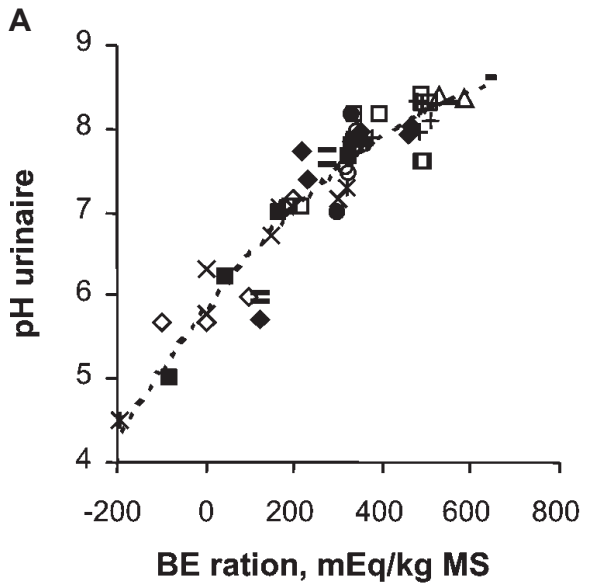

acido-basique et permet de quantifier l'intensité des réponses en fonction du modèle animal étudié. Les courbes obtenues permettent aussi de quantifier l'intensité des mécanismes, mécanismes décrits jusqu'ici d'un point de vue physiologique uniquement. Ainsi, les $\mathrm{pH}$ du sang et de l'urine augmentent lorsque le BE de la ration s'élève jusqu'à $400 \mathrm{mEq} / \mathrm{Kg} \mathrm{MS}$, traduisant le fait que les cations apportés par la ration sont échangés contre les protons, permettant ainsi la lutte contre l'acidose. $\mathrm{Au}$-delà d'un $\mathrm{BE}$ de la ration de 350 $\mathrm{mEq} / \mathrm{Kg} \mathrm{MS}$, les $\mathrm{pH}$ restent stables : l'animal ne lutte pas contre l'acidose mais contre l'alcalose liée à une arrivée trop massive de cations, donc à une élimination de protons importante.

Les mécanismes de régulation développés par les animaux pour lutter contre les variations de $\mathrm{pH}$ sanguin sont

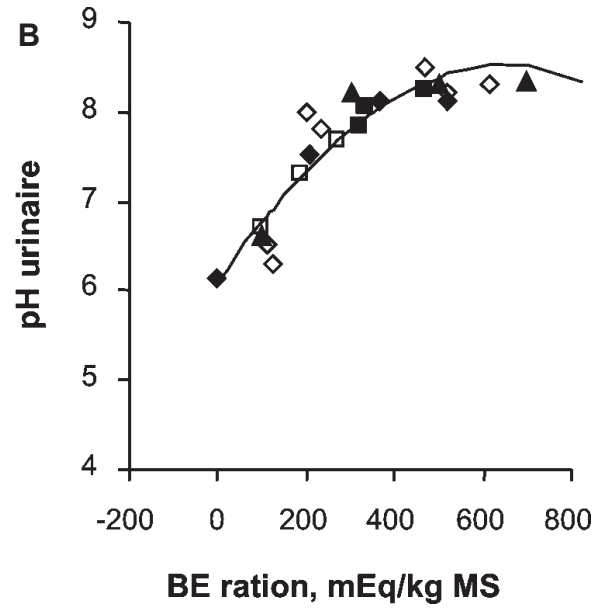

capables de lutter contre de fortes baisses de pH sanguin, tandis qu'ils n'admettent que de très légères hausses. Ainsi, le $\mathrm{pH}$ et les concentrations en bicarbonates du sang, tout comme le $\mathrm{pH}$ urinaire peuvent diminuer de manière notable lorsque le $\mathrm{BE}$ de la ration est faible. A l'inverse, lorsque le $\mathrm{BE}$ de la ration augmente au-delà de $250 \mathrm{mEq} / \mathrm{kg} \mathrm{MS}$, le $\mathrm{pH}$ et les concentrations en bicarbonates du sang ne varient plus que dans de très faibles proportions. Parallèlement, le $\mathrm{pH}$ urinaire ne dépasse pas 8,6 unités, seulement 1,2 unité au dessus du pH normal du sang, tandis qu'il peut descendre jusqu'à 4,1 lorsque le $\mathrm{BE}$ de la ration est faible. Il semble en fait que le sang et l'urine saturent très rapidement face à un excès de bases. Cette observation laisse supposer qu'une trop forte augmentation du $\mathrm{BE}$ de la ration pourrait entraîner un état d'alcalose métabo- lique non compensée, les animaux devenant incapables d'excréter l'excès de cations. Ce phénomène contribue à expliquer la gravité de l'alcalose métabolique ainsi que la baisse d'ingestion parfois observée avec des $\mathrm{pH}$ sanguins élevés.

Si les lois de réponse sont très proches entre les espèces, il est néanmoins possible de dégager quelques différences entre elles. Ainsi, le pH et surtout les concentrations en bicarbonates du sang sont plus sensibles aux variations du $\mathrm{BE}$ de la ration chez les porcs. Lorsque le $\mathrm{BE}$ de la ration augmente de - 200 à $+400 \mathrm{mEq} / \mathrm{kg} \mathrm{MS}$, le $\mathrm{pH}$ sanguin augmente de 0,12 unité $\mathrm{pH}$ chez le porc contre 0,10 chez la vache laitière et 0,07 chez le ruminant en croissance (tableau 4). Dans le même temps, les concentrations en bicarbonates augmentent de $10,0 \mathrm{mEq} / \mathrm{L}$ chez le porc et de seulement $6,5 \mathrm{mEq} / \mathrm{L}$ pour tous les ruminants. Ces différences inter spécifiques pourraient être liées au rôle tampon que joue le rumen. En effet, la plupart des sels utilisés pour augmenter le $\mathrm{BE}$ de la ration (notamment les bicarbonates de sodium et de potassium) participent aussi à tamponner le milieu ruminal (Erdman 1988, cf. § 3.3). Une autre explication possible est que les ruminants disposent d'une réserve de bicarbonates très importante constituée par les bicarbonates présents dans le rumen dont ceux prélevés dans le sang lors de l'absorption des Acides Gras Volatils (AGV).

La $\mathrm{P}_{\mathrm{CO} 2}$ est peu affectée par le $\mathrm{BE}$ de la ration chez les vaches laitières (figure 4). Elle s'accroît linéairement avec le $\mathrm{BE}$ de la ration, mais dans des marges étroites. Elle varie de 40,6 à

Figure 4. Effet de l'accroissement du $B E$ de la ration sur la $P_{\mathrm{CO} 2} d u$ sang des vaches en lactation $\left(\boldsymbol{A}\right.$. $P_{\mathrm{CO} 2}=41,25+0,0034$ ' $B E$ $\left.r^{2}=0,96, E T R=1,216, P<0,05, n=51\right)$, des ruminants en croissance $\left(B\right.$. $P_{C O 2}=35,2+0,0101^{\prime} B E-9,46^{\prime} 10^{-6} 6^{\prime} B E^{2}, r^{2}=0,94$, $E T R=1,662 P<0,10, \mathrm{n}=23)$ et des porcs en croissance et finition $\left(\boldsymbol{C}\right.$. $P_{\mathrm{CO} 2}=70.0+0,0241^{\prime} B E-6,18^{\prime} 10^{-5}{ }^{\prime} B E^{2}, r^{2}=0,95$, $E T R=3,073, P<0,01, \mathrm{n}=46)$.

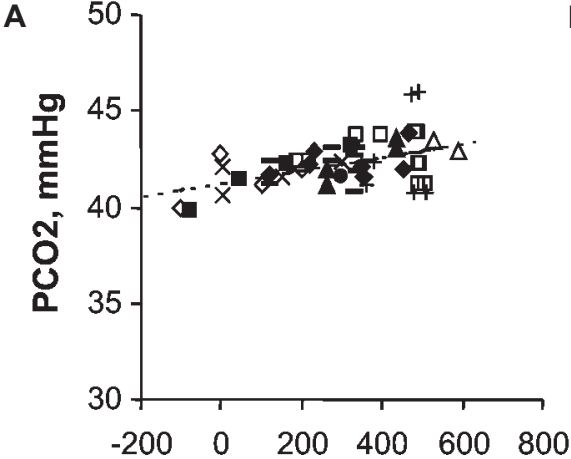

BE ration, $\mathrm{mEq} / \mathrm{kg} \mathrm{MS}$

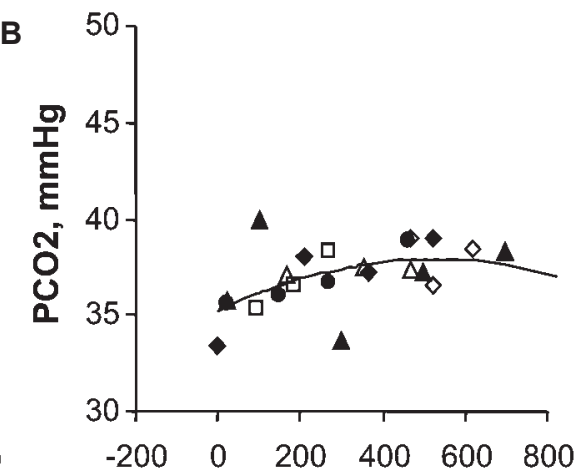

BE ration, $\mathrm{mEq} / \mathrm{kg}$ MS

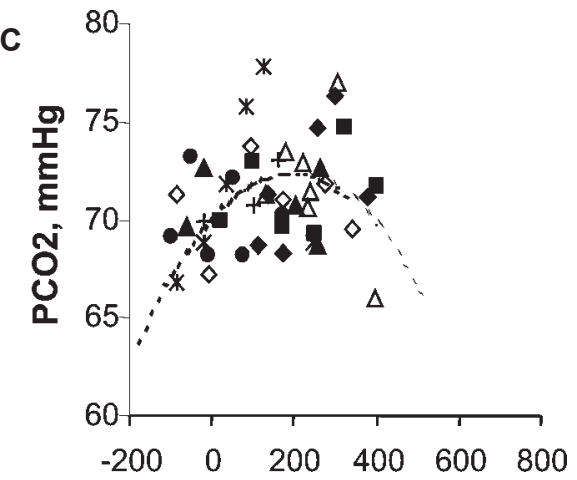

$\mathrm{BE}$ ration, $\mathrm{mEq} / \mathrm{kg} \mathrm{MS}$ 
Figure 5. Effet de l'accroissement du BE de la ration sur la DIF du sang (DIF $\left.=\mathrm{Na}^{+}+\mathrm{K}^{+}-\mathrm{Cl}^{-}\right)$des vaches en lactation $(\boldsymbol{A}$. DIF $=$ $\left.42,02+0,0164^{\prime} B E, r^{2}=0,89, E T R=2,408, P<0,0001, n=49\right)$, des ruminants en croissance $\left(B\right.$. DIF $=31.80+0,0106^{\prime} B E$ $r^{2}=0,99, E T R=2,308, P<0,005, \mathrm{n}=23$ ) et des porcs en croissance et finition (C. DIF $=47.12+0,0122{ }^{\prime} B E, r^{2}=0,62$, $E T R=3,342, P<0,05, \mathrm{n}=30)$.

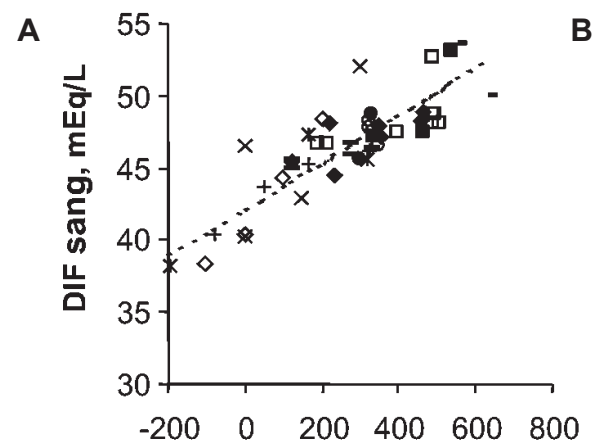

$\mathrm{BE}$ ration, $\mathrm{mEq} / \mathrm{kg} \mathrm{MS}$

B

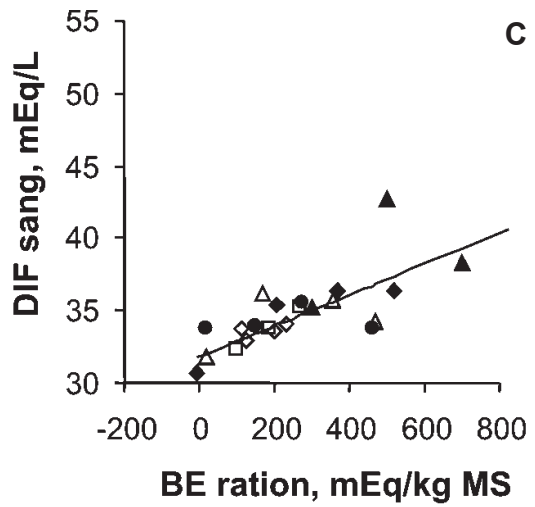

2.4 / Des réponses de l'équilibre ionique du sang très semblables entre les ruminants en croissance et les monogastriques

Le BE de la ration affecte logiquement l'équilibre ionique du sang. La différence en ions forts du sang (DIF) s'accroît linéairement avec l'accroissement du BE chez tous les animaux, l'effet étant d'ampleur comparable selon la base de données considérée (figure 5). En moyenne, un accroissement du $\mathrm{BE}$ de la ration de - 200 à $+400 \mathrm{mEq} / \mathrm{kg} \mathrm{MS}$ correspond à un accroissement de la différence en ions forts du sang de $9 \mathrm{mEq} / \mathrm{L}$ chez la vache laitière, de 7 chez les ruminants en croissance et le porc. La natrémie et la kaliémie ne sont pas affectées par le BE de la ration malgré des variations très importantes d'apports en sodium et en potassium par la ration. En revanche, la chlorémie diminue linéairement avec l'accroissement du $\mathrm{BE}$ de la ration chez tous les types d'animaux, l'effet étant très semblable quelle que soit la base de données considérée (figure 6). En

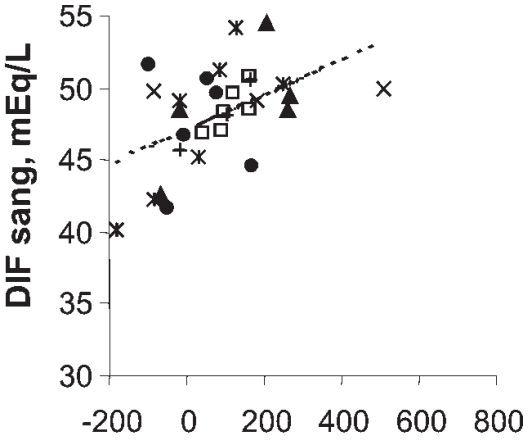

$B E$ ration, $\mathrm{mEq} / \mathrm{kg} \mathrm{MS}$ moyenne, un accroissement du BE de la ration de -200 à $+400 \mathrm{mEq} / \mathrm{Kg}$ MS correspond à une diminution de la chlorémie d'environ $6 \mathrm{mEq} / \mathrm{L}$.

L'étude bibliographique confirme l'implication du chlore dans le maintien de l'homéostasie de l'animal. En effet, les concentrations en sodium et en potassium du sang n'ont pas été affectées par l'accroissement du BE de la ration, même si ce sont ces ions qui ont servi à augmenter le BE. Cette absence de fluctuation témoigne d'une régulation stricte de leurs teneurs. En revanche, la chlorémie sanguine a systématiquement diminué avec l'accroissement du BE de la ration, et cela de manière très marquée quel que soit le modèle étudié. Cet effet est certainement à mettre en relation avec le fait que cet ion est échangé contre le bicarbonate pour maintenir l'électroneutralité du sang. D'ailleurs, chez la vache laitière, il faut noter que dans la gamme de BE envisagée, les effets de l'accroissement du BE sur la chlorémie (- $6 \mathrm{mEq} / \mathrm{L})$ et sur les concentrations en ici

Figure 6. Effet de l'accroissement du BE de la ration sur la concentration en $\mathrm{Cl}^{-}$du sang des vaches en lactation $(\boldsymbol{A}$. [Cl'] $=104,4$ - 0,0105 $\left.\mathrm{B} E, r^{2}=0,62, E T R=2,772, P<0,01, \mathrm{n}=49\right)$, des ruminants en croissance $\left(B\right.$. $\left[C I^{-}\right]=108,7-0,0077^{\prime} B E, r^{2}=0,99, E T R$ $=1,495, P<0,01, \mathrm{n}=23)$ et des porcs en croissance et finition $\left(\boldsymbol{C} . \mathrm{Cl}^{-}\right]=103,4-0,0096{ }^{\prime} B E, r^{2}=0,44, E T R=3,900, P=0,10$, $\mathrm{n}=30)$.

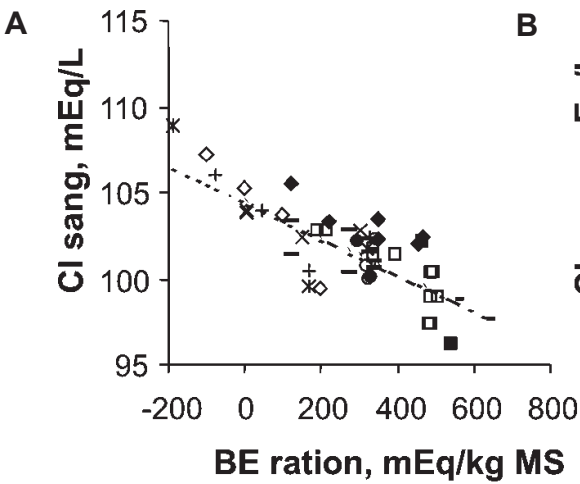

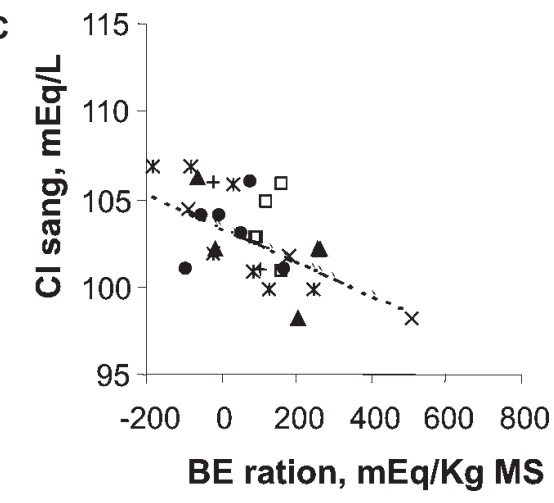


Figure 7. Relation obtenue entre la chlorémie du sang et la teneur en $\mathrm{HCO}_{3}^{-}$. $\left[\mathrm{HCO}_{3}{ }^{-}\right]=110,49-0,859{ }^{\prime}\left[\mathrm{Cl}^{-}\right], \mathrm{n}=40$, $E T R=1,123, R^{2}=0,84$ (d'après ApperBossard 2005).

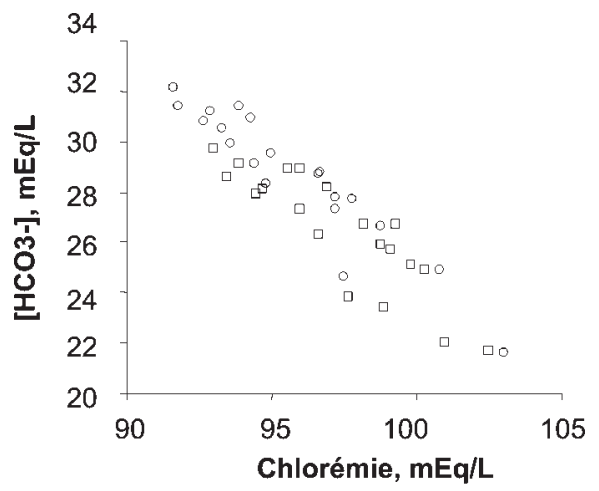

Avant repas $\square$ Après repas

bicarbonates $(+6,5 \mathrm{mEq} / \mathrm{L})$ sont très cohérents avec un échange mole par mole de chlore et de bicarbonates pour maintenir l'électroneutralité des fluides et des cellules (figure 7). Ces résultats montrent que lors de l'apport d'ions par la ration, le chlore est le principal ion impliqué dans les variations de la DIF du sang et finalement dans la modulation de l'homéostasie acido-basique du sang. D'autres travaux avaient suggéré que les variations de la chlorémie n'étaient pas uniquement liées à la variation des apports, mais aussi à des processus physiologiques. Ainsi, Tucker et al (1988a), en étudiant l'effet de l'accroissement du $\mathrm{BE}$ de la ration obtenu par modulation soit du sodium, soit du potassium, soit du chlore, ont rapporté systématiquement une baisse de la chlorémie sanguine avec l'accroissement du BE de la ration quel que soit l'ion utilisé.

\section{3 / Quantification de l'effet de la composition en ions de la ration sur l'ingestion et les performances zootech- niques des monogastriques et des ruminants}

Afin de quantifier les effets de la variation de la composition en ions de la ration sur les réponses zootechniques, nous avons travaillé sur les bases de données des vaches en lactation et des porcs en croissance et finition décrites dans la partie 2.1. Les données sur les ruminants en croissance étaient trop peu nombreuses et n'étaient pas assez homogènes pour effectuer une analyse quantitative pertinente. Les variables testées sont : la Matière Sèche Ingérée (MSI), la production laitière (PL), les Taux Butyreux (TB) et Protéique (TP), la production laitière à $4 \%$ (PL4\%), les productions en Matières Grasses (MG) et en Matières Protéiques (MP) du lait chez les vaches en lactation et la MSI et le Gain Moyen Quotidien (GMQ) chez le porc. La MSI des vaches a varié de 13 à $21 \mathrm{~kg} \mathrm{MS/j}$ (après correction de l'effet publication) et leur PL a varié de 21 à $27 \mathrm{~kg} / \mathrm{j}$. La MSI des porcs a varié de 1 à $1,6 \mathrm{~kg} / \mathrm{j}$, le GMQ était relativement faible, compris entre 0,35 et $0,72 \mathrm{~kg} / \mathrm{j}$.

\section{1 / La MSI augmente avec l'ac- croissement du BE de la ration}

Chez les deux espèces, l'accroissement du BE de la ration accroît fortement la MSI (figure 8). Lorsque le BE de la ration augmente de -200 à +400 $\mathrm{mEq} / \mathrm{kg} \mathrm{MS}$, la MSI augmente de $6,4 \mathrm{~kg} / \mathrm{j}$ (soit $35 \%$ ) chez les vaches laitières et de $0,42 \mathrm{~kg} / \mathrm{j}$ (soit $29 \%$ ) chez les porcs. La relation est curvilinéaire, l'effet du BE étant surtout important lorsqu'il s'accroît depuis des valeurs négatives vers des valeurs légèrement positives. Ainsi, l'accroissement du BE de - 200 à + 100 mEq/kg MS s'accompagne d'un accroissement de la MSI de $4,7 \mathrm{~kg}$ chez la vache et de $0,41 \mathrm{~kg}$ chez le porc. L'ingestion maximale est obtenue pour un $\mathrm{BE}$ de la ration plus faible chez le porc que chez la vache en lactation (respectivement à 200 et à $400 \mathrm{mEq} / \mathrm{kg} \mathrm{MS}$ ). Au-delà de ces valeurs, l'ingestion est stable, voire diminue légèrement. D'ailleurs, Roche et al (2003) ont rapporté une légère chute de l'ingestion chez des vaches laitières avec un $\mathrm{BE}$ de la ration dépassant $500 \mathrm{mEq} / \mathrm{kg}$ MS. Chez le porc, en se basant sur l'équation obtenue, l'ingestion passe de $1,43 \mathrm{~kg} / \mathrm{j}$ à $1,37 \mathrm{~kg} / \mathrm{j}$, soit une baisse de 4,5\%. L'accroissement de

Figure 8. Effet de l'accroissement du BE de la ration sur les quantités de matières sèches ingérées (MSI) des vaches en lactation (A. $\left.M S I=15,81+0,014^{\prime} B E-1,69.10^{-5}{ }^{\prime} B E^{2}, r^{2}=0,81, E T R=0,996, P<0,05, n=55\right)$ et des porcs en croissance et finition (B. $M S I=1,29+0,0012^{\prime} B E-2,52 \cdot 10^{-6} \cdot B E^{2}, r^{2}=0.98, E T R=0,097, P<0,0001, \mathrm{n}=58$ ).

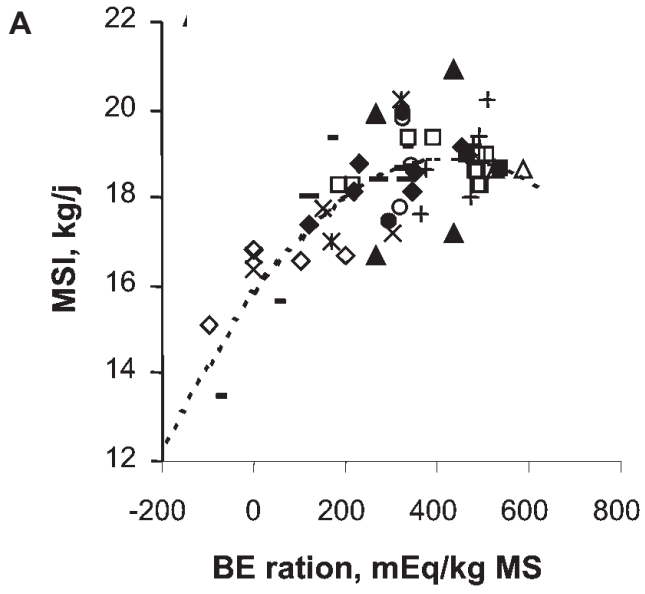

- Coppock et al 1979

+ Delaquis et Block 1995b

- Kilmer et al 1980

$\diamond$ Tucker et al 1988a

Tucker et al 1991a et b

$\times$ Waterman et al 1991

- West et al 1992
\ Tucker et al 1994

- West et al 1991
B

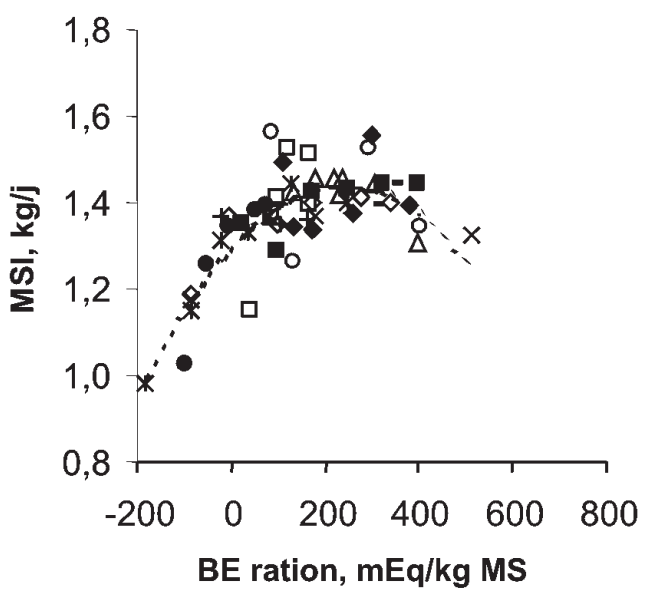

- Haydon et al 1990

$\triangle$ Wondra et al 1995

- Patience et Wolynetz 1990 exp 1 * Patience et Wolynetz 1990 exp 2

+ Patience et Chaplin 1997

- Patience et al 1987, exp 2

$\diamond$ Patience et al 1987, exp 4

- Patience et al 1987, exp1

- Patience et al 1987, exp 3

$\square$ Honeyfield et Froseth 1985 
Figure 9. Effet de l'accroissement du $B E$ de la ration sur la production laitière $(P L)$ des vaches en lactation $(\boldsymbol{A} . P L=22,80+0,009$ $\left.B E-1,15.10^{-5} \cdot B E^{2}, E T R=1,519, r^{2}=0.94, P<0,05, n=55\right)$ et le gain moyen quotidien (GMQ) des porcs en croissance et finition (B. $\left.G M Q=0,53+0,0007^{\prime} B E-1,56.10^{-6}{ }^{\prime} B E^{2}, r^{2}=0.93, E T R=0,062, P<0,0001, \mathrm{n}=58\right)$.

A

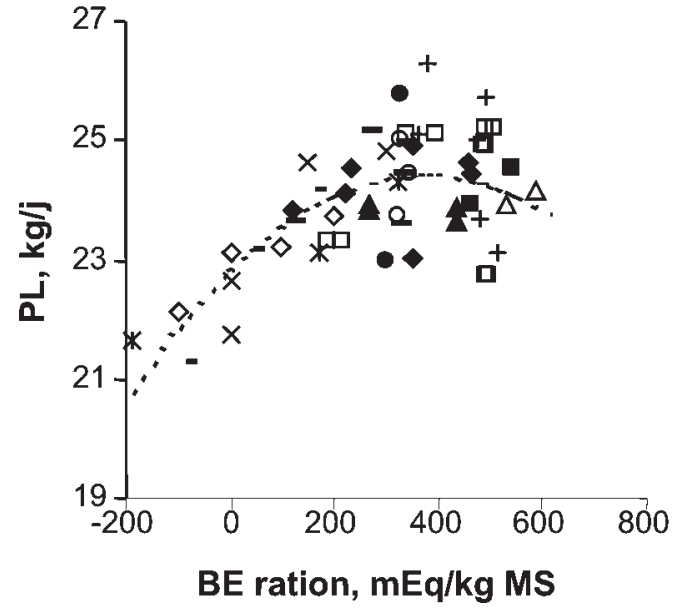

B

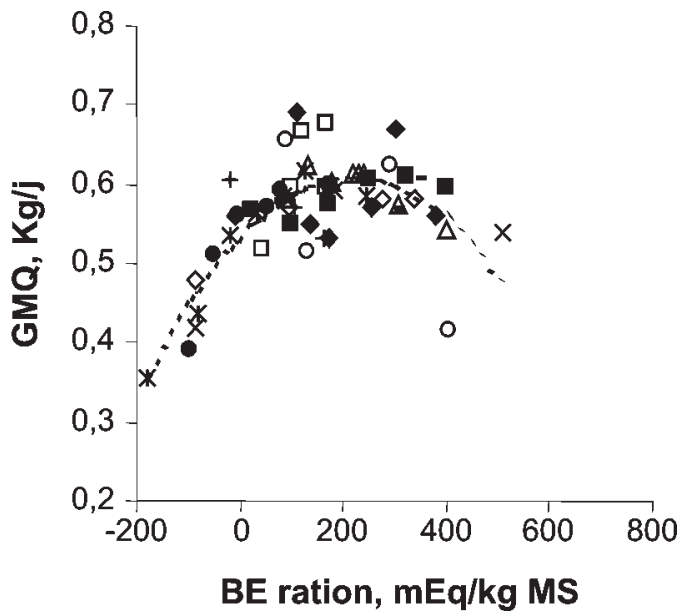

l'ingestion parallèlement à celui du $\mathrm{BE}$ de la ration se retrouve également chez les ruminants en croissance (Jackson et al 1992, Ross et al 1994a et b) et les agneaux (Fauchon et al 1994). Chez les bovins en croissance, des valeurs très élevées du BE de la ration semblent également réduire légèrement l'ingestion (Jackson et al 1992).

\section{2 / Les performances de pro- duction augmentent avec l'ac- croissement du bilan électroly- tique}

L'accroissement du BE de la ration est associé à une stimulation de la production du lait. Comme pour l'ingestion, la réponse est maximale pour un $\mathrm{BE}$ de $400 \mathrm{mEq} / \mathrm{kg} \mathrm{MS}$, elle est surtout nette lorsque le BE s'accroît depuis des valeurs négatives (figure 9). Toutefois, l'effet sur le lait est moins net et plus variable que celui sur les quantités ingérées puisque une augmentation du $\mathrm{BE}$ de la ration de - 200 à + $400 \mathrm{mEq} / \mathrm{kg} \mathrm{MS}$ accroît la production de $4,02 \mathrm{~kg}$ soit seulement $16 \%$. L'accroissement du BE accroît également la production de matières protéiques et de matières grasses principalement du fait de son effet sur le volume du lait (figure 10). Le TP n'est pas affecté et le TB non plus tant que le $\mathrm{BE}$ reste inférieur à $300 \mathrm{mEq} / \mathrm{kg} \mathrm{MS}$. Pour des BE plus élevés, le TB tend à s'accroître (tableau 5). Cette augmentation pourrait être relié au fait que l'augmentation du BE de la ration est obtenue par adjonction de sels cationiques, notamment des bicarbonates de sodium et de potassium qui ont pu contribuer à tamponner le milieu ruminal et donc à stabiliser les fermentations ruminales.
L'augmentation du BE permet aussi d'améliorer le GMQ chez des porcs. Comme pour l'ingestion, la réponse est maximale pour un BE proche de 200 $\mathrm{mEq} / \mathrm{kg}$ MS et elle est surtout nette lorsque le BE s'accroît depuis des valeurs négatives (figure 9). Cet effet sur le GMQ est très important et est moins variable que l'effet obtenu sur la PL des vaches en lactation. Une augmentation du BE de la ration de -200 à + $400 \mathrm{mEq} / \mathrm{Kg}$ MS accroît le GMQ de $0,23 \mathrm{~kg} / \mathrm{j}$ (soit 40\%). Finalement l'augmentation du BE accroît aussi l'efficacité de la production puisque l'indice de consommation diminue de 2,9 à 2,3 lorsque le BE s'accroît de - 200 à 400 $\mathrm{mEq} / \mathrm{kg} \mathrm{MS}$.

L'analyse de données a permis de dégager des lois de réponse générales des performances zootechniques de vaches en pleine lactation et de porcs

Figure 10. Effet de l'accroissement du BE de la ration sur la production de matières grasses (MG, figure $A . M G=791+0,288^{\prime} B E$, $E T R=68,4, r^{2}=0,88, P<0,01, \mathrm{n}=53$ ) et protéiques (MP, figure $B . M P=717+0,306^{\prime} B E-3,44.10^{-4}{ }^{\prime} B E^{2}, E T R=34,7, r^{2}=$ $0,97, P<0,05, \mathrm{n}=53$ ) du lait des vaches.

A

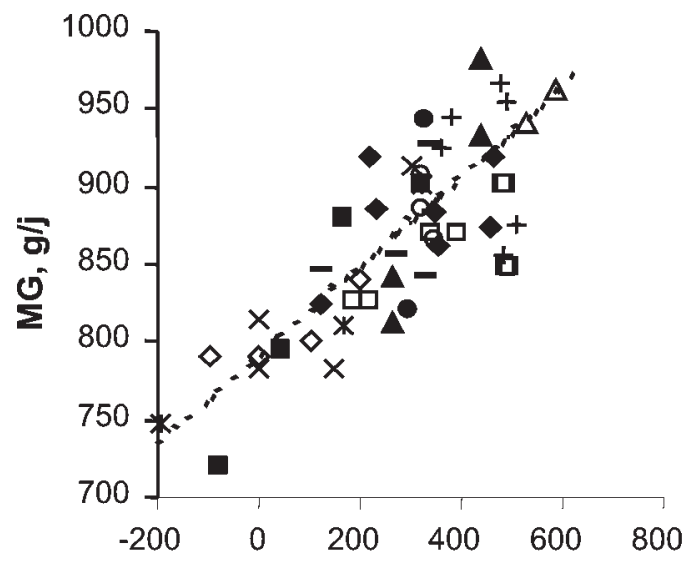

BE ration, $\mathrm{mEq} / \mathrm{kg} \mathrm{MS}$
B

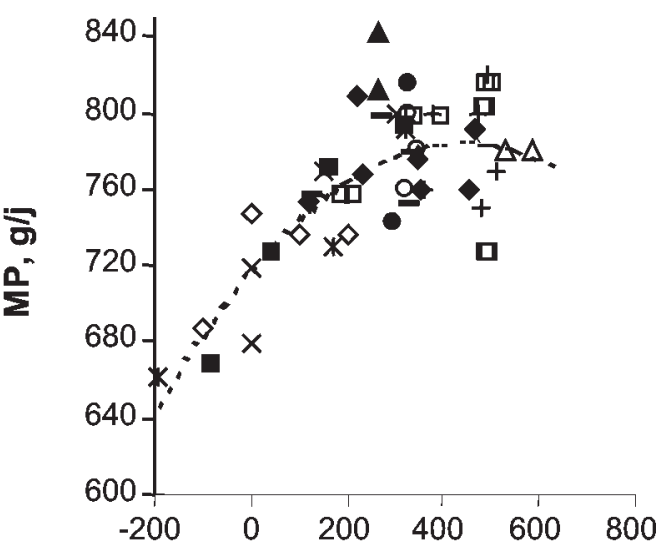

BE ration, $\mathrm{mEq} / \mathrm{kg} \mathrm{MS}$ 
Tableau 5. Coefficients de régression entre le $B E$ de la ration $\left(B E=\mathrm{Na}^{+}+\mathrm{K}^{+}-\mathrm{Cl}^{-}\right.$.) et les réponses zootechniques obtenus dans les modèles vaches en lactation et porcs en croissance et finition.

\begin{tabular}{|c|c|c|c|}
\hline Variable & $\mathrm{BE}=0$ & L & $\mathbf{Q}$ \\
\hline \multicolumn{4}{|l|}{ Vaches en lactation } \\
\hline$M S I^{1}, \mathbf{k g} / \mathbf{j}$ & 15,8 & 0,014 & $-1,69.10^{-5^{* * *}}$ \\
\hline Production Laitière, $\mathrm{kg} / \mathrm{j}$ & 22,8 & $0,009^{*}$ & \\
\hline Production Laitière $4 \%, \mathrm{~kg} / \mathrm{j}$ & 21,1 & $0,006^{*}$ & NS \\
\hline Taux Butyreux, g/kg & 35,2 & NS & $1,70.10^{-5^{* *}}$ \\
\hline Taux Protéique, g/kg & & NS & NS \\
\hline Matières Grasses, $\mathrm{g} / \mathrm{j}$ & 791 & $0,288^{* *}$ & NS \\
\hline Matières Protéiques, $\mathrm{g} / \mathrm{j}$ & 717 & 0,306 & $3,44.10^{-4^{*}}$ \\
\hline \multicolumn{4}{|l|}{ Porcs en croissance } \\
\hline $\begin{array}{l}\mathrm{MSI}^{1}, \mathrm{~kg} / \mathrm{j} \\
\mathrm{GMQ}^{2}, \mathrm{~kg} / \mathrm{i}\end{array}$ & $\begin{array}{l}1,293 \\
0,532\end{array}$ & $\begin{array}{l}0,0012 \\
0,0007^{* * *}\end{array}$ & $\begin{array}{l}-2,5.10^{-} \\
-1,6.10^{-6+*}\end{array}$ \\
\hline
\end{tabular}

$1 \mathrm{MSI}$ : Matière Sèche Ingérée.

$2 \mathrm{GMQ}=$ Gain Moyen Quotidien.

$\dagger \mathrm{P}<0,10$

${ }^{*} \mathrm{P}<0,05$

${ }^{* *} P<0,01$

${ }^{* * *} P<0,001$

en croissance face à un accroissement $\mathrm{du} \mathrm{BE}$ de la ration. Cette analyse constitue une première étape pour établir des conseils en alimentation. Ainsi, elle établit clairement qu'un apport élevé de cations est nécessaire pour maximiser les performances de vaches laitières fortes productrices et de porcs en croissance. Un tel besoin témoigne de la nécessité accrue qu'ont ces animaux, au métabolisme très intense, d'éliminer les protons et d'avoir une quantité importante de bicarbonates. L'analyse permet ainsi de faire des recommandations pour des vaches laitières fortes productrices, nourries avec des rations comportant 40 à $70 \%$ de concentré et pour des porcs nourris avec des rations riches en glucides. Avec de telles rations, un $\mathrm{BE}$ de 300 à $400 \mathrm{mEq} / \mathrm{kg}$ MS permet d'optimiser l'ingestion et la production laitière. Dans une synthèse récente, Meschy (2007) a proposé un apport de $250 \mathrm{mEq} / \mathrm{kg} \mathrm{MS}$ pour des vaches en lactation. Ainsi, en terme de recommandations, il semble que $250 \mathrm{mEq} / \mathrm{kg} \mathrm{MS}$ soit un minimum, $400 \mathrm{mEq} / \mathrm{kg} \mathrm{MS}$ un maximum pour optimiser les performances des vaches en lactation.

Pour faire des recommandations précises et adaptées, la nature et la quantité des autres composants de la ration doivent être pris en compte, notamment la teneur en concentré et/ou en protéines. En effet, des travaux récents (Apper Bossard et al 2006) ont montré que l'augmentation du $\mathrm{BE}$ de la ration permettait d'accroître les quantités ingérées et la production de lait $4 \%$ dans le cas des rations riches en concentré (et donc plus acidogènes) alors qu'aucun effet n'a été observé dans le cas de rations riches en fourrage. De même, l'effet de l'accroissement du BE de la ration sur l'ingestion apparaît plus important pour des rations pauvres en protéines que pour des rations bien pourvues. En particulier, Apper-Bossard (2005) a montré que l'accroissement du $\mathrm{BE}$ de la ration augmentait la MSI des vaches en lactation avec une ration à faible teneur en PDIE $(77 \mathrm{~g} / \mathrm{kg})$ mais pas avec une ration contenant $107 \mathrm{~g}$ $\mathrm{PDIE} / \mathrm{kg}$. Ainsi, pour élaborer un système de prévision des risques des chutes de performances chez le ruminant, il sera nécessaire de considérer l'équilibre entre les facteurs de risques et de sécurisation, avec, pour variable d'ajustement de l'animal, l'ingestion volontaire. A notre connaissance de telles études n'ont pas été réalisées chez le porc et il serait intéressant par exemple de déterminer si les protéines alimentaires constituent un moyen de sécurisation d'une ration riche en glucides.

Les lois de réponses zootechniques face à un accroissement du BE de la ration nécessitent aussi d'être modulées en fonction du stade physiologique de l'animal. Ainsi, chez la vache laitière, si un apport élevé de cations est nécessaire en début et en pleine lactation, il a été montré chez les vaches autour du part qu'un apport excédentaire en anions permettait de diminuer la fréquence d'apparition de la fièvre vitulaire (Jorgensen 1974, Horst 1986). La fièvre vitulaire se caractérise par une hypocalcémie sévère avec une concentration plasmatique inférieure à
1,25 mmol/L (Tauriainen 2001), la norme étant comprise entre 2,25 et $2,50 \mathrm{mmol} / \mathrm{L}$ en début de lactation. Cette maladie se déclenche à la parturition, suite à l'augmentation brutale de la demande en calcium avec le début de lactation. Cette demande accrue de calcium ne peut être couverte par les seules réserves de calcium plasmatique et extracellulaire et par les apports alimentaires. Plusieurs études ont montré qu'une ration de tarissement avec un BE faible entraîne une augmentation de la calcémie, ce qui diminue la fréquence d'apparition de la fièvre vitulaire (Goff et Horst 1998, Thilsing-Hansen et al 2002). Cet effet est d'autant plus marqué que la teneur en calcium de la ration est élevée (Horst 1986). La valeur du $\mathrm{BE}$ de la ration qui permet de réduire au maximum les risques est située entre +50 et $+150 \mathrm{mEq} / \mathrm{kg} \mathrm{MS}$ (Horst et al 1997), ce qui est très différent des recommandations proposées pour des vaches laitières en pleine lactation.

\section{3 / Voies d'action de l'effet du $B E$ de la ration sur les perfor- mances zootechniques : un effet systémique marqué}

La forte similitude observée entre les réponses zootechniques des porcs en croissance et des vaches en lactation face à l'accroissement du BE de la ration suggère une voie d'action systémique et pas seulement une action au niveau du rumen. Effectivement, dans tous les modèles, les réponses de l'ingestion sont très proches de celles du $\mathrm{pH}$ et des concentrations en bicarbonates sanguins. En particulier, les valeurs $\mathrm{du} \mathrm{BE}$ de la ration pour lesquelles la MSI est maximale sont très proches des valeurs de BE de la ration pour lesquelles les concentrations en bicarbonates et le $\mathrm{pH}$ du sang sont les plus forts. Ces observations supportent l'hypothèse que les améliorations des réponses zootechniques peuvent être liées à une diminution de l'acidité dans le sang des animaux recevant une ration au $\mathrm{BE}$ très positif et dont l'activité métabolique est intense. Toutefois, il n'y a pas, à notre connaissance de démonstration expérimentale du phénomène.

Les sels de bicarbonates utilisés pour augmenter le BE de la ration sont des sels qui ont un effet tampon au niveau du rumen (Erdman 1988). Dès lors, il est légitime de penser que l'ajout de ces sels a pu améliorer le fonctionnement du rumen et améliorer les performances zootechniques des ruminants. Néanmoins, la comparaison entre les 
ruminants et les monogastriques met en évidence des réponses systémiques finalement très similaires, laissant supposer que l'effet observé au niveau systémique peut jouer un rôle dans l'amélioration des performances aussi bien chez le monogastrique que chez le ruminant. Par ailleurs, Apper-Bossard (2005) a rapporté une absence de modification de la teneur en acétate et en propionate du rumen avec un accroissement du BE de la ration. De la même façon, d'autres travaux ont rapporté des effets limités de l'accroissement du BE de la ration sur les produits terminaux de la fermentation, même lorsque des sels de bicarbonate étaient ajoutés à la ration (Tucker et al 1988a, Ross et al 1994b, La Manna et al 1999), suggérant un effet modéré sur le fonctionnement ruminal.

Néanmoins, l'étude ne permet pas d'exclure un effet de l'accroissement du $\mathrm{BE}$ de la ration sur le fonctionnement du rumen. La moindre importance des phénomènes compensatoires mis en place chez le ruminant, la réponse moins importante et plus variable obtenue sur la PL par rapport à celle obtenue sur le GMQ peuvent peut-être s'expliquer en partie par le rumen. En effet, si l'effet de l'accroissement du $\mathrm{BE}$ de la ration est prépondérant au niveau systémique, plusieurs études montrent que l'apport de cations limite la chute du $\mathrm{pH}$ ruminal (Ross et al 1994a et b, Apper-Bossard 2005). Par ailleurs, les effets des sub- stances tampons (notamment du bicarbonate de sodium ou de potassium) sur le $\mathrm{pH}$ ruminal sont assez bien établis. Meschy et al (2004) ont montré qu'un apport de $1 \%$ de bicarbonate de sodium accroît le $\mathrm{pH}$ ruminal de 0,07 unité et la MSI de $0,5 \mathrm{~kg} / \mathrm{j}$. Or, dans cette étude bibliographique, les sels retenus pour augmenter les quantités de sodium et de potassium ont précisément été, pour majorité du bicarbonate de sodium ou de potassium. Ainsi, une partie des sels a pu servir de tampon au niveau ruminal.

\section{Conclusion}

Ce travail a permis de quantifier les effets de l'accroissement du BE de la ration sur l'homéostasie acido-basique et sur les performances zootechniques des animaux à forts besoins et de dégager des lois de réponse. Dans tous les cas, l'accroissement du $\mathrm{BE}$ de la ration se traduit par une augmentation du $\mathrm{pH}$ et des teneurs en bicarbonates du sang, une hausse du $\mathrm{pH}$ sanguin et une augmentation de la MSI et des performances zootechniques. Ces résultats mettent en évidence l'importance de maîtriser l'apport ionique dans la ration. Il reste toutefois nécessaire d'affiner ce travail afin de pouvoir donner des recommandations adaptées à l'animal, en fonction de son stade physiologique et des autres caractéristiques de la ration.
En ce qui concerne les mécanismes, nous avons montré que l'accroissement $\mathrm{du} \mathrm{BE}$ de la ration permet de neutraliser des quantités plus importantes de protons et finalement de limiter les besoins d'excrétion urinaire d'acides volatils (d'où l'accroissement du $\mathrm{pH}$ urinaire avec l'augmentation du BE de la ration) lorsque les animaux sont nourris avec une ration acidogène et ont un métabolisme accru. La comparaison ruminants vs monogastriques permet d'établir que l'effet du BE est systémique même si l'on ne peut écarter, dans le cas des ruminants un effet au niveau du rumen. La plupart des études a utilisé des sels qui ont un pouvoir tampon connu au niveau ruminal et qui permettent un accroissement du recyclage de tampons salivaires suite à l'accroissement des teneurs en bicarbonates du sang.

D'un point de vue pratique, la très bonne relation obtenue entre l'accroissement du BE de la ration et l'évolution du $\mathrm{pH}$ urinaire est un résultat intéressant. En effet, en affinant cette loi de réponse, notamment en fonction des autres composants de la ration et de l'état de santé des animaux (les infections urinaires pouvant être des facteurs de variation de $\mathrm{pH}$ à prendre en compte), le $\mathrm{pH}$ urinaire pourrait devenir un indicateur stable du BE de la ration.

\section{Références}

Apper-Bossard E., 2005. Equilibre de la ration et homéostasie acido-basique chez la vache laitière forte productrice : rôle de la balance alimentaire en cations et en anions, de la teneur en protéines et en énergie rapidement dégradable. Thèse, Université Rennes I, 150p.

Apper-Bossard E., Peyraud J.L., Faverdin P., Meschy F., 2006. Changing dietary cationaniondifference for dairy cows fed with two contrasting levels of concentrate in diets. J. Dairy Sci., 89, 749-760.

Beighle D.E., Tucker W.B., Hemken R.W., 1988. Interactions of dietary cation anion balance and phosphorus: effects on growth and serum inorganic phosphorus in dairy calves. J. Dairy Sci., 71, 3362-3368.

Bernard C., 1859. Leçons sur les propriétés physiologiques et les altérations pathologiques des liquides de l'organisme. Baillère J.B. et fils (Eds), Paris, France.

Block E., 1994. Manipulation of dietary cationanion difference on nutritionally related production diseases, productivity, and metabolic responses of dairy cows. J. Dairy Sci., 77, 1437-1450.

Block E., 1995. Anioncation balance and its effects on performance of ruminants. Rec. Adv. Anim. Nutr., 163-179.
Brugère H., 2001. Equilibre et régulation acido-basique dans l'organisme. Rôles de l'ion bicarbonate. In : Journée Solvay, le bicarbonate de sodium chez les ruminants, 1-14.

Bushinsky D.A., Chabala J.M., Gavrilov K.L., Levi-Setti R., 1999. Effects of in vivo metabolic acidosis on midcortical bone ion composition. Am. J. Physiol., 277, F813-F819.

Bushinsky D.A., Smith S.B., Gavrilov K.L., Gavrilov L.F., Li J., Levi-Setti R., 2003. Chronic acidosis-induced alteration in bone bicarbonate and phosphate. Am. J. Physiol., 285, F532-F539.

Ching S.V., Fettman M.J., Hamar D.W. Nagode L.A., Smith K.R., 1989. The effect of chronic dietary acidification using ammonium chloride on acid-base status metabolism in the adult cat. J. Nutr., 119, 902-915

Constable P.D., 1999. Clinical assessment of acid-base status: strong ion difference theory. Vet. Clin. North Am. Food Anim. Pract., 15, 447-471.

Coppock C.E., Aguirre R.A., Chase L.E., Lake G.B., Oltenacu E.A., McDowell R.E., Fettman M.J., Woods M.E., 1979. Effect of a low chloride diet on lactating Holstein cows. J. Dairy Sci., 62, 723-731.

Davenport H.W., 1971. ABC de l'équilibre biochimique acido-basique. Masson et Cie (Ed), Paris, France.
Delaquis A., Block E., 1995a. The effects of changing ration ingredients on acid-base status, renal function, and macromineral metabolism. J. Dairy Sci., 78, 2024-2039.

Delaquis A., Block E., 1995b. Dietary cationanion difference, acid-base status, minera metabolism, renal function and milk production of lactating cows. J. Dairy Sci., 78, 2259-2284.

Den Hartog L.A., Schamp T.A.G., Mors R.A.B., Verstegen M.W.A., Van Gils L.G.M., 1989. The effect of dietary electrolyte balance on blood parameters and the performance of veal calves. Livest. Prod. Sci., 21, 213-222.

Dersjant-Li Y., 2000. Impact of dietary cation anion difference in fish and pigs: a comparative study. Thèse, Wageningen Institute of Animal. Science, Pays-Bas, 169p.

Erdman R.A., 1988. Dietary buffers requirements of the lactating dairy cows: a review. J. Dairy Sci., 71, 3246-3266.

Escobosa A., Coppock C.E., Rowe Jr L.D., Jenkins W.L., Gates C.E., 1984. Effects of dietary sodium bicarbonate and calcium chloride on physiological responses of lactating dairy cows in hot weather. J. Dairy Sci., 67, 574-584.

Fauchon C., Seoane J.R., Bernier J.F., 1995. Effects of dietary cation-anion concentrations on 
performance and acid-base balance in growing lambs. Can. J. Anim. Sci., 75, 145-151.

Faverdin P., Bareille N., Vérité R., 1999. Effects of rumen energy supply timing on feed intake control in lactating dairy cows. J. Dairy Sci., 82, 2443-2454.

Fredeen A.H., DePeters E.J., Baldwin R.L., 1988. Characterization of acid-base disturbances and effects on calcium and phosphorus balances of dietary fixed ions in pregnant or lactating does. J. Anim. Sci., 66, 159-173.

Frick K.K., Bushinsky D.A., 1998. Chronic metabolic acidosis reversibly inhibits extracellular matrix gene expression in mouse osteoblasts. Am. J. Physiol., 275, F840-F847.

Goff J.P., Horst R.L., 1998. Use of hydrochloric acid as a source of anions for prevention of milk fever. J. Dairy Sci., 81, 2874-2880.

Goff J.P., Horst R.L., Mueller F.J., Miller J.K Kiess J. A., Dowle H.H., 1991. Addition of chloride to a prepartal diet high in cations increases 1,25-Dihydroxyvitamin D response to hypolcalcemia preventing in milk fever. J. Dairy Sci., 74, 3863-3871.

Haydon K.D., West J.W., McCarter M.N., 1990. Effect of dietary electrolyte balance on performance and blood parameters of growingfinishing swine fed in high ambient temperatures. J. Anim. Sci., 68, 2400-2406.

Honeyfield D.C., Froseth J.A., 1985. Effects of dietary sodium and chloride on growth, efficiency of feed utilization, plasma electrolytes and plasma basic amino-acids in young pigs. J. Nutr., 115, 1366-1371.

Horst R.L., 1986. Regulation of calcium and phosphorus homeostasis in the dairy cow. J. Dairy Sci., 69, 604-616.

Horst R.L., Goff J.P., Reinhardt T.A., Buxton D.R., 1997. Strategies for preventing milk fever in dairy cattle. J. Dairy Sci., 80, 1269-1280.

Jackson J.A., Hopkins D.M., Xin Z., Hemken R.W., 1992. Influence of cation-anion balance on feed intake, body weight gain, and humoral response of dairy calves. J. Dairy Sci., 75, 1281-1286.

Jorgensen N.A., 1974. Combating milk fever. J. Dairy Sci., 57, 933-944.

Kilmer L.H., Muller L.D., Wangsness P.J., 1980. Addition of sodium bicarbonate to rations of per- and postpartum dairy cows. J. Dairy. Sci., 63, 2026-2036

Krieger N.S., Bushinsky D.A., Frick K.K., 2003. Cellular mechanism of bone resorption induced by metabolic acidosis. Semin. Dialys, $16,463-466$.

La Manna A.F., Owens F.N., Janloo S., Ahmad Y.H., Welty S.D., 1999. Impact of dietary cation anion balance on water intake and physiological measurements of feedlot cattle. Anim. Sci. Res. Rep., 152-158

McKinnon J.J., Christensen D.A., Laarveld B., 1990. The influence of bicarbonate buffers on milk production and acid-base balance in lactating dairy cows. Can. J. Anim. Sci., 70, 875-886.

Meghji S., Morrison M.S., Henderson B., Arnett T.R., 2001. pH dependence of bone resorption: mouse calvarial osteoclasts are activated by acidosis. Am. J. Physiol., 280, E112E119.

Meschy F., 2007. Alimentation minérale et vitaminique des ruminants : actualisation des connaissances. INRA Prod. Anim., 2, 119-128.
Meschy F., Guéguen L., 1998. Les recommandations d'apport alimentaire en éléments minéraux : analyse et perspective. Renc. Rech. Rum., 5, 235-240.

Meschy F., Bravo D., Sauvant D., 2004 Analyse quantitative des réponses des vaches laitières à l'apport de substances tampon. INRA Prod. Anim., 17, 11-18.

Mongin P., 1981. Recent advances in dietary cation-anion balance: application in poultry. Proc. Nutr. Soc., 40, 285-294.

Mueller R.K. Topliff D.R., Freeman D.W. McAllister C., Carter S.D., Cooper S.R., 1999. Effect of varying DCAD on the acid-base status of mature sedentary horses with varying starch source and level of intake. Anim. Sci. Res. Rep., 189-193.

Murakami A.E., Oviedo-Rondo E.O., Martins E.N., Pereira M.S., Scapinello C., 2001. Sodium and chloride requirements of growing broilers chicken fed corn-soybean diets. Poult. Sci., 80, 289-294.

Oetzel G.R., Fettman M.J., Hamar D.W. Olson J.D., 1991. Screening of anionic salts for palatability, effects on acid-base status and urinary calcium excretion in dairy cows. J. Dairy Sci., 74, 965-971.

Patience J.F., Wolynetz F., 1990. Influence of dietary undetermined anion on acid-base status and performance in pigs. J. Nutr., 120, 579-587.

Patience J.F., Chaplin R.K., 1997. The relationship among dietary undetermined anion, acid-base balance, and nutrient metabolism in swine. J. Anim. Sci., 75, 2445-2452.

Patience J.F., Austic R.E., Boyd R.D., 1987. Effect of dietary electrolyte balance on growth and acid-base status in swine. J. Anim. Sci., 64, 457-466

Peyraud J.L., Apper-Bossard E., 2006. L'acidose latente chez la vache laitière. INRA Prod. Anim., 19, 79-92.

Roche J.R., Petch S., Kay J.K., 2003. Dietary cation-anion difference and the health and production of pasture-fed dairy cows. 1 . Dairy cows in early lactation. J. Dairy Sci., 86, 970-978.

Ross J.G., Spears J.W., Garlich J.D., 1994a. Dietary electrolyte balance effects on performance and metabolic characteristics in growing steers. J. Anim. Sci., 72, 1842-1848.

Ross J.G., Spears J.W., Garlich J.D., 1994b. Dietary electrolyte balance effects on performance and metabolic characteristics on finishing steers. J. Anim. Sci., 72, 1600-1607.

Sauveur B., Mongin P., 1978. Tibial dyschondroplasia, a cartilage abnormality in poultry. Ann. Biol. Anim. Bioch. Biophys., 18, 87-98.

Shapiro B.A., Harrison R.A., Cane R.D. Templin R., 1992. Gaz du sang. Applications cliniques. Frison-Roche (Ed), Paris, France, 395p.

Spanghero A., 2004. Prediction of urinary and blood $\mathrm{pH}$ in non lactating dairy cows fed anionic diets. Anim. Feed Sci. Technol., 116,83-92.

Stewart P.A., 1978. Independent and dependent variables of acid-base control. Resp. Physiol., 33, 9-26.

Stewart P.A., 1983. Modern quantitative acidbase chemistry. Can. J. Physiol. Pharmacol., 61, 1444-1461.

Takagi H., Block E., 1991. Effects of manipulating dietary cation-anion balance on macromineral balance in sheep. J. Dairy Sci., 74 , 4202-4214.
Tauriainen S., 2001. Dietary cation-anion balance and calcium and magnesium intake of the dry cows. PhD Thesis University of Helsinski, Finland, 53p.

Thilsing-Hansen T., Jorgensen N.A. Ostergaard S., 2002. Milk fever control principles: a review. Acta Vet. Scand., 43, 1-19.

Tucker W.B., Hogue J.F., 1990. Influence of sodium chloride or potassium chloride on systemic acid base status, milk yield and mineral metabolism in lactating dairy cows. J. Dairy Sci. 73, 3485-3493

Tucker W.B., Harrison G.A., Hemken R.W., 1988a. Influence of dietary cation-anion balance on milk, blood, urine, and rumen fluid in lactating dairy cattle. J. Dairy Sci., 71, 346-354.

Tucker W.B., Xin Z., Hemken R.W., 1988b. Influence of dietary calcium chloride on adaptative changes in acid-base status and minera metabolism in lactating dairy cows fed a diet high in sodium bicarbonate. J. Dairy Sci., 71, 1587-1597.

Tucker W.B., Hogue J.F., Waterman D.F. Swenson T.S., Xin Z., Hemken R.W., Jackson J.A., Adams G.D., Spicer L.J., 1991a. Role of sulfur and chloride in the dietary cation-anion balance equation for lactating dairy cattle. J. Anim. Sci., 69, 1205-1213.

Tucker W.B., Jackson J.A., Hopkins D.M., Hogue J.F., 1991b. Influence of dietary Sodium bicarbonate on the Potassium metabolism of growing dairy calves. J. Dairy Sci., 74, 22962302

Tucker W.B., Shin I.S., Hogue J.F., Aslam M., Adams G.D., Van Koevering M.T., Vernon, R.K., Cummings K.R., 1994. Natural sodium sesquicarbonate fed for an entire lactation: influence on performance and acid-base status. J. Dairy Sci., 77, 3111-3117.

Van Slyke D.D., 1922.On the measurement of buffer values and on the relationship of buffe value to the dissociation constant of the buffer and the concentration and reaction of the buffer solution. J. Biol. Chem., 52, 525-570.

Wall D.L., Topliff D.R., Freeman D.W. Wagner D.G., Breazile J.W., Stutz W.A., 1992. Effect of dietary cation-anion balance on urinary mineral excretion in exercised horses. J. Equine Vet. Sci., 12, 168-171.

Wamberg S., Engel K., Stigsen P., 1985. Acidbase balance in ruminating calves given sodium hydroxide-treated straw. Brit. J. Nutr., 54, 655-667.

Waterman D.F., Swenson T.S., Tucker W.B., Hemken R.W., 1991. Role of magnesium in the dietary cation-anion balance equation for ruminants. J. Dairy Sci., 74, 1866-1873

West J.W., Mullinix B.G., Sandifer T.G., 1991. Changing dietary electrolyte balance for dairy cows in cool and hot environment. J. Dairy Sci. 71, 1662-1674.

West J.W., Haydon K.D., Mullinix B.G., Sandifer T.G., 1992. Dietary cation-anion balance and cation source effects on production and acid-base status of heat-stressed cows. J. Dairy Sci., 75, 2776-2786.

Whitehead C.C., 1997. Dyschondroplasia in poultry. Proc. Nutr. Soc., 56, 957-966.

Wondra K.J., Hancock J.D., Behnke K.C., Hines R.H.., 1995. Effects of dietary buffers on growth performance, nutrient digestibility and stomach morphology in finishing pigs. J. Anim. Sci., 73, 414-420. 


\section{Résumé}

La maîtrise de l'alimentation des animaux à fort niveau de production est nécessaire pour éviter des pertes économiques importantes. Parmi les pistes explorées, le bilan électrolytique, défini comme [sodium] + [potassium]-[chlore], est une voie intéressante pour limiter la production d'acides à l'origine de troubles métaboliques. A partir de trois bases de données (vaches en lactation, porcs en croissance et ruminants en croissance), cette synthèse étudie par analyse de covariance, les réponses zootechniques et celles de l'homéostasie acidobasique en fonction de la valeur du bilan électrolytique de la ration. Les quantités ingérées, la production de lait à $4 \%$ et le gain moyen quotidien augmentent avec l'augmentation du bilan électrolytique. Dans le même temps, le pH et les concentrations en bicarbonates du sang suivent la même évolution. La chlorémie diminue fortement avec l'augmentation du bilan électrolytique : le chlore est l'ion le plus impliqué dans la régulation de l'homéostasie acido-basique. Enfin, le pH urinaire augmente avec l'accroissement du bilan électrolytique de la ration, la corrélation obtenue est très forte. La voie urinaire est donc la principale voie d'excrétions des ions, et le pH urinaire pourrait être un bon indicateur de la valeur du bilan électrolytique des rations distribuées.

L'augmentation du bilan électrolytique de la ration est donc un moyen efficace de lutter contre la production d'acides liée au métabolisme intense des animaux à fort potentiel de production. L'homogénéité des résultats entre les différents modèles suggère un effet du bilan électrolytique à un niveau systémique, même si quelques différences entre ruminants et monogastriques laissent à penser un effet au niveau du rumen.

\section{Abstract}

\section{Effects of dietary cation-anion difference on performance and acid-base status: a review}

Improving food practices of high producing animals is required to avoid important economic losses. The dietary anion-cation difference, described as [sodium] + [potassium]-[chlorine], should aid in preventing metabolic disorders caused by acid production. From three databases (dairy cows, growing pigs and growing ruminants), performance and acid-base status responses on an increase of dietary cationanion difference were studied by a covariance analysis. Intake, $4 \%$ fat corrected milk and average daily gain increased with increasing dietary cation-anion difference. At the same time, blood $\mathrm{pH}$ and blood bicarbonate concentration increased whereas blood chlorine decreased, suggesting that chlorine is the main ion implicated in the acid-base regulation. The urinary pH increased with increasing dietary cation-anion difference and it was highly correlated with the dietary cation-anion difference. Renal excretion was the main way to excrete ions and urinary $\mathrm{pH}$ seemed to be a good predictor of dietary cation-anion difference. The results suggest that an increased dietary cation-anion difference could prevent metabolic disorders due to an intense metabolism. Moreover, the results suggest that the main way to improve performances is based on a systemic level, even though a rumen effect could explain the difference between ruminants and pigs.

APPER-BOSSARD E., PEYRAUD J.-L., DOURMAD J.-Y., 2009. Effet du bilan électrolytique de la ration sur l'équilibre acido-basique et les performances zootechniques des animaux domestiques à fort niveau de production. Inra Prod. Anim., $22,117-130$ 